\title{
Ghost-free theories with arbitrary higher-order time derivatives
}

\author{
Hayato Motohashi, ${ }^{a, 1}$ Teruaki Suyama $^{b}$ and Masahide Yamaguchi $^{b}$ \\ ${ }^{a}$ Center for Gravitational Physics, Yukawa Institute for Theoretical Physics, \\ Kyoto University, \\ Kyoto 606-8502, Japan \\ ${ }^{b}$ Department of Physics, Tokyo Institute of Technology, \\ 2-12-1 Ookayama, Meguro-ku, Tokyo 152-8551, Japan \\ E-mail: hayato.motohashi@yukawa.kyoto-u.ac.jp, \\ suyama@phys.titech.ac.jp, gucci@phys.titech.ac.jp
}

ABSTRACT: We construct no-ghost theories of analytic mechanics involving arbitrary higher-order derivatives in Lagrangian. It has been known that for theories involving at most second-order time derivatives in the Lagrangian, eliminating linear dependence of canonical momenta in the Hamiltonian is necessary and sufficient condition to eliminate Ostrogradsky ghost. In the previous work we showed for the specific quadratic model involving third-order derivatives that the condition is necessary but not sufficient, and linear dependence of canonical coordinates corresponding to higher time-derivatives also need to be removed appropriately. In this paper, we generalize the previous analysis and establish how to eliminate all the ghost degrees of freedom for general theories involving arbitrary higher-order derivatives in the Lagrangian. We clarify a set of degeneracy conditions to eliminate all the ghost degrees of freedom, under which we also show that the Euler-Lagrange equations are reducible to a second-order system.

Keywords: Black Holes, Classical Theories of Gravity

ARXIV EPRINT: 1804.07990

\footnotetext{
${ }^{1}$ Corresponding author.
} 


\section{Contents}

1 Introduction 1

2 Examples 3

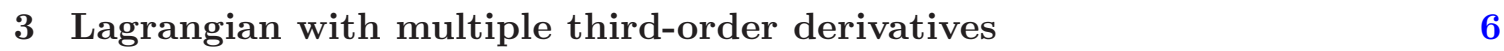

$\begin{array}{lll}3.1 & \text { Hamiltonian analysis } & 7\end{array}$

$\begin{array}{ll}3.2 & \text { Euler-Lagrange equation } \\ \end{array}$

4 Lagrangian with arbitrary higher-order derivatives $\quad 14$

$\begin{array}{ll}4.1 \text { Hamiltonian analysis } & 15\end{array}$

$\begin{array}{lll}4.2 & \text { Euler-Lagrange equation } & 22\end{array}$

5 Conclusions and discussion $\quad 24$

$\begin{array}{ll}\text { A Lagrangian with single third-order derivative } & 25\end{array}$

\section{Introduction}

The presence of inflation and the current accelerated expansion of the Universe is strongly supported by observational results such as the cosmic microwave background radiation anisotropies [1-4] and type Ia supernovae $[5,6]$. One simple way to explain these two regimes of accelerated expansion is to introduce additional degrees of freedom (DOFs) to General Relativity and modify the law of gravitation. In general, if one adds higher-thanfirst-order derivative terms to an action, it leads to ghost DOFs known as Ostrogradsky ghost [7]. Of course, even if there is ghost DOF in a theory, it would not be problematic as long as it appears above the scale one is interested in. However, in cosmology one sometimes considers a situation, in which higher derivative terms play dominant roles in the dynamics. In such a case, the effective theory view point would be invalidated and ghost DOFs must be removed to guarantee healthiness and/or predictability of the theory. One of such famous examples is Horndeski theory [8] (equivalent to generalized Galileon $[9,10]$ ), which is the most general single-field scalar-tensor theory whose Euler-Lagrange equations of motion (EOMs) are up to second-order in derivatives, and thus free from additional ghost DOFs.

It was recognized recently that the requirement of second-order Euler-Lagrange equations is too strong to avoid ghost DOFs [11-13]. This is because the highest orders of derivatives in the Euler-Lagrange equations do not necessarily give a correct number of initial conditions: even if Euler-Lagrange equations directly derived from an action a priori include higher-order time-derivative terms, there is no ghost instability as long as they can 
be recast into second-order system without introducing extra variables. Ghost-free condition can be thus investigated in a more explicit way in the Hamiltonian picture. Under the assumption that a Lagrangian involves multiple variables and all the variables have up to $n$-th order derivatives $(n \geq 2)$, the Ostrogradsky theorem states that if the Lagrangian is nondegenerate with respect to the highest order derivatives, the Hamiltonian is unbounded due to the existence of ghost DOFs, which develops instabilities when the system couples to normal systems [7].

One may then expect that the Ostrogradsky ghost can be removed by requiring the degeneracy of Lagrangian with respect to the highest-order derivatives, which corresponds to a removal of the highest $2 n$-th order derivatives in the Euler-Lagrange equation. However, evading the Ostrogradsky theorem is not sufficient to construct healthy models (i.e. no ghosts) as it is just a statement of the sufficient condition for the existence of ghosts that non-degeneracy with respect to the highest-order derivatives inevitably leads to ghosts. In other words, degeneracy with respect to the highest-order derivatives does not guarantee the absence of all ghost DOFs. In fact, it was demonstrated in [14] that there exists a class of Lagrangians with up to $n$-th order derivatives that satisfies the degeneracy with respect to the highest-order derivative but ends up with unbounded Hamiltonian due to the ghost DOFs associated with $(2 n-1)$-th order derivatives in the Euler-Lagrange equation. Definitely, one needs more degeneracy conditions to eliminate all the ghost DOFs. Another important point is that it is necessary for application to general theories of modified gravity to go beyond the assumption that all the variables have the same order of derivatives in Lagrangian, and to consider Lagrangian with several types of variables with different orders of derivatives. With several types of variables of different orders of derivatives in Lagrangian, degeneracy conditions are more nontrivial.

In [15], the degeneracy condition was clarified for a specific Lagrangian for the quadratic-order model of degenerate higher-order scalar-tensor (DHOST) theories, which involves "regular" variables with at most first-order derivative and single "special" variable with at most second-order derivatives. The degeneracy condition for general Lagrangian with multiple regular and special variables was developed in [16]. The degeneracy condition derived in [16] applies to any model involving up to second-order derivative terms in time. Indeed, to the best of our knowledge, all of theories of modified gravity discussed so far include only up to second-order derivative terms in time. Specifically, Horndeski derived the most general second-order Euler-Lagrange equations for single-field scalar-tensor theory, and then reconstructed the action starting from an action with arbitrary finite order derivative terms, but the obtained action includes only up to second-order derivatives [8]. Gao proposed another extension, which involves arbitrary higher-order derivatives in space but up to second-order derivatives in time [17]. Theories beyond Horndeski $[12,13]$ and degenerate higher-order scalar-tensor theories $[15,18]$ also involve up to second-order derivatives. Thus, as far as we know, there is no explicit example in the context of field theory, which includes third (or even higher) order derivatives in time but can avoid ghost instabilities. ${ }^{1}$

\footnotetext{
${ }^{1}$ It should be noted that some of infinite-order derivative (non-local) theories can avoid ghost instabilities at least classically. For example, see refs. [19, 20] and references therein.
} 
In the previous work [21], we provided a specific model which is quadratic in variables and involves third-order time derivatives in the Lagrangian. Our finding is that elimination of the canonical momenta in the Hamiltonian by the constraints and degeneracy conditions does not kill all the ghost DOFs associated with the higher derivatives and the ghost DOFs still remain. Although the remaining ghost DOFs are hidden in a very nontrivial way in the Hamiltonian, in the case of the quadratic model, canonical transformation makes those ghost DOFs manifest themselves as linear terms of canonical coordinates [21]. Presence of additional ghosts not in the form of the linear terms of the canonical momenta is a crucial difference from theories involving at most second-order time derivatives. We derived in [21] a set of degeneracy conditions for the quadratic model, and confirmed that the Hamiltonian equations as well as the Euler-Lagrange equations are reducible to a system of second-order differential equations when the degeneracy conditions are imposed.

In this paper, we further generalize the previous analysis for theories involving at most second-order derivatives performed in [16] (see also [22] for a similar analysis, [23] for a case including fermionic degree of freedom, and [15, 18, 24-27] for field theoretical extensions), as well as the previous analysis for the specific theory involving third-order derivatives in [21]. Since the degeneracy conditions obtained in [21] only apply to the specific quadratic model involving at most third-order derivatives, in the present paper, we first clarify a set of degeneracy conditions for general Lagrangian involving third-order derivatives. We also confirm that the Euler-Lagrange equations can be reduced into a second order differential equations. Furthermore, we consider general Lagrangian involving arbitrary higher-order derivatives, and derive a set of degeneracy conditions, under which we confirm that the Euler-Lagrange equations are reducible into second-order system. Our result applies to any form of Lagrangian involving any higher-order derivatives. Thus, it is an important first step for construction of ghost-free theories of modified gravity with third- and even higher-order derivatives.

The organization of the rest of the paper is as follows. In section 2 we provide an explicit example which includes arbitrary higher-order derivatives in a Lagrangian but does not have Ostrogradsky ghosts. In section 3, we investigate general Lagrangian involving three set of multiple variables with at most first-, second-, and third-order derivatives, respectively. We derive a set of conditions to avoid Ostrogradsky ghosts. We show that with these conditions the Euler-Lagrange equations are reducible to second-order system. Some of them are satisfied identically for single variable case, which is supplemented in the appendix. Finally in section 4 we extend our analysis to general Lagrangian with arbitrary finite higher-order derivatives. Section 5 is devoted to conclusions and discussion.

\section{Examples}

The specific example of ghost-free theory of quadratic model involving third-order derivatives is presented in [21]. In this section we provide an example of ghost-free theory involving arbitrary finite higher-order derivatives in Lagrangian. We show that the EulerLagrange equations are rearrangeable to second-order system, and that through the Hamiltonian analysis the system does not possess the Ostrogradsky ghosts. 
We consider the following Lagrangian

$$
L=\frac{1}{2} \frac{\dot{q}^{2}}{1+\phi^{(d+1)}}+\frac{1}{2} \dot{\phi}^{2},
$$

where $q=q(t), \phi=\phi(t)$, and $\phi^{(d+1)}$ represents the $(d+1)$-th derivative of $\phi(t)$ with $d \geq 1$ being an integer. This model is a generalization of the toy model considered in section 7.1 of [28], which corresponds to $d=1$ case. The Euler-Lagrange equations for $q$ and $\phi$ are given by

$$
\begin{aligned}
\frac{d}{d t}\left(\frac{\dot{q}}{1+\phi^{(d+1)}}\right) & =0, \\
\ddot{\phi}+(-1)^{d+1} \frac{1}{2} \frac{d^{d+1}}{d t^{d+1}}\left(\frac{\dot{q}^{2}}{\left(1+\phi^{(d+1)}\right)^{2}}\right) & =0 .
\end{aligned}
$$

Despite the appearance of higher derivative terms, we can see this system of equations is actually second-order system as follows. From the first equation we note that $\frac{\dot{q}}{1+\phi^{(d+1)}}=$ const. Plugging it to the second equation, we obtain $\ddot{\phi}=0$, with which the first equation reduces to $\ddot{q}=0$. Therefore the system is equivalent to

$$
\ddot{q}=\ddot{\phi}=0,
$$

which is clearly a second-order system for 2 variables $q, \phi$ and requires 4 initial conditions for $\{q, \dot{q}, \phi, \dot{\phi}\}$. It is straightforward to consider a generalization of the model where the Lagrangian is given by a sum of (2.1) for multiple $q, \phi$ variables with different orders of derivatives.

Let us check the number of DOFs and the absence of Ostrogradsky ghost for the system (2.1) by Hamiltonian analysis. By introducing auxiliary variables $Q^{i}$ and Lagrange multipliers $\lambda_{i}$, we rewrite the Lagrangian $L$ in (2.1) to an equivalent form

$$
\begin{aligned}
L_{\mathrm{eq}} & =L\left(\dot{q}, \dot{Q}^{d}, Q^{1}\right)+\sum_{i=0}^{d-1} \lambda_{i}\left(\dot{Q}^{i}-Q^{i+1}\right), \\
L\left(\dot{q}, \dot{Q}^{d}, Q^{1}\right) & =\frac{1}{2} \frac{\dot{q}^{2}}{1+\dot{Q}^{d}}+\frac{1}{2}\left(Q^{1}\right)^{2},
\end{aligned}
$$

where we define $Q^{0} \equiv \phi$. This Lagrangian yields at most second-order EOMs for $2(d+$ 1) variables, $\left\{q, Q^{d}, Q^{i}, \lambda_{i}\right\}$ with $i=0, \cdots, d-1$. Thus, a priori this system requires $4(d+1)$ initial conditions. The form of $L_{\text {eq }}$ allows us to define the canonical momenta for $\left\{q, Q^{d}, Q^{i}, \lambda_{i}\right\}$ in the standard way:

$$
p=L_{\dot{q}}=\frac{\dot{q}}{1+\dot{Q}^{d}}, \quad P_{d}=L_{\dot{Q}^{d}}=-\frac{1}{2}\left(\frac{\dot{q}}{1+\dot{Q}^{d}}\right)^{2}, \quad P_{i}=\lambda_{i}, \quad \rho_{i}=0 .
$$

The last two equations are primary constraints associated with the introduction of auxiliary variables. In addition to them, we note that there is an additional primary constraint $P_{d}=F(p) \equiv-p^{2} / 2$. In total, the primary constraints are

$$
\Phi_{i} \equiv P_{i}-\lambda_{i} \approx 0, \quad \bar{\Phi}_{i} \equiv \rho_{i} \approx 0, \quad \Psi \equiv P_{d}-F \approx 0 .
$$


Time evolution of the canonical variables is governed by the total Hamiltonian, which is given by

$$
\begin{aligned}
H_{T} & =H+\mu_{i} \Phi_{i}+\bar{\mu}_{i} \bar{\Phi}_{i}+\nu \Psi \\
H & =H_{0}+\sum_{i=0}^{d-1} P_{i} Q^{i+1} \\
H_{0} & =\dot{Q}^{d} P_{d}+\dot{q} p-L,
\end{aligned}
$$

where $\mu_{i}, \bar{\mu}_{i}, \nu$ are Lagrange multipliers. The linear terms $\sum P_{i} Q^{i+1}$ correspond to the Ostrogradsky ghosts, by which the Hamiltonian is unbounded.

Since the primary constraints need to be satisfied through time evolution, we require time derivative of the primary constraints remain vanishing as consistency condition. From the consistency condition $\dot{\bar{\Phi}}_{i} \approx 0$ and $\dot{\Phi}_{i} \approx 0$, we respectively obtain

$$
\mu_{i} \approx 0, \quad \bar{\mu}_{i} \approx\left\{\Phi_{i}, H\right\}+\nu\left\{\Phi_{i}, \Psi\right\}
$$

The consistency condition for $\Psi$ is given by

$$
0 \approx \dot{\Psi}=\{\Psi, H\}+\nu\{\Psi, \Psi\}
$$

Needless to say, the last term vanishes identically, but we kept it for later convenience. Actually, the fact that this term identically vanishes means that this system satisfies the second degeneracy condition [see (3.20)]. From (2.10) we obtain a secondary constraint

$$
-\{\Psi, H\}=P_{d-1} \approx 0
$$

We then check the consistency condition $0=\dot{P}_{d-1}=\left\{P_{d-1}, H\right\}+\nu\left\{P_{d-1}, \Psi\right\}$ and obtain a tertiary constraint $P_{d-2}=0$. Actually, it is clear from the linear terms $\sum P_{i} Q^{i+1}$ in the Hamiltonian that we successively obtain the constraints

$$
P_{d-1} \approx 0, \quad P_{d-2} \approx 0, \quad, \cdots, \quad P_{1} \approx 0
$$

Finally the consistency condition for $P_{1}=0$ gives

$$
0 \approx \dot{P}_{1}=P_{0}-Q_{1}
$$

which is the last constraint as its consistency condition is identically satisfied. Clearly, the constraints remove linear terms in the Hamiltonian, and thus eliminate the Ostrogradsky ghosts.

Hence we expect the system possesses only healthy 2 DOFs. To count the number of DOFs, we classify all the constraints obtained above to first class and second class by checking the Poisson brackets between them, which form the Dirac matrix. The Dirac 
matrix is given by

\begin{tabular}{l|ccccc} 
& $\Phi_{j}$ & $\bar{\Phi}_{j} \Psi$ & $P_{d-1} \cdots$ & $P_{1}$ & $P_{0}-Q_{1}$ \\
\hline$\Phi_{i}$ & 0 & $-\mathbf{1}$ & & & \\
$\bar{\Phi}_{i}$ & $\mathbf{1}$ & 0 & & & \\
$\Psi$ & & & & & \\
$P_{d-1}$ & & & & & \\
$\vdots$ & & & & \\
$P_{1}$ & & & 0 & 1 \\
$P_{0}-Q_{1}$ & & & & -1 & 0
\end{tabular}

where $\mathbf{1}$ is the unit matrix and blank arguments are zeros. Hence we end up with $2 d+2$ second class constraints $\Phi_{i}, \bar{\Phi}_{i}, P_{1}, P_{0}-Q_{1}$, and $d-1$ first class constraints $\Psi, P_{d-1}, P_{d-2}, \cdots, P_{2}$. Starting the primary first class constraint $\Psi$, we can check the Dirac test is satisfied: since the chain of the Poisson brackets exhausts all first class constraints as $\{H, \Psi\}=P_{d-1},\left\{H, P_{d-1}\right\}=P_{d-2}, \cdots,\left\{H, P_{3}\right\}=P_{2}$, all the first class constraints are generator of gauge transformations. Therefore, the number of DOFs for the system is given by $[4(d+1)-(2 d+2)-2(d-1)] / 2=2$, which is consistent with the Euler-Lagrange picture.

\section{$3 \quad$ Lagrangian with multiple third-order derivatives}

The example in section 2 shows that it is indeed possible to involve arbitrary higherorder derivatives in Lagrangian and construct no-ghost theory. In this case, some part of degeneracy conditions could be identically satisfied due to the particular form of the Lagrangian. For more general Lagrangians, we need to impose a certain set of degeneracy conditions, for which it is worthwhile to remind the lesson obtained in [21]. In [21], we investigated the quadratic model involving third-order derivatives and clarified that it is necessary to impose a sufficient number of degeneracy conditions to eliminate all ghost DOFs. In particular, fixing linear terms in conjugate momenta in the Hamiltonian is not sufficient as linear terms in canonical coordinates themselves lurk in the Hamiltonian in a nontrivial way. We need to impose degeneracy conditions and continue the Dirac algorithm until we are left with healthy DOFs whose number matches that of variables. The final goal of the present paper is to generalize this process for general Lagrangian involving arbitrary higher-order derivatives (see section 4).

In this section, we consider Lagrangian involving multiple variables $\psi^{n}(t)$ with thirdorder derivatives and multiple regular variables $q^{i}(t)$ :

$$
L\left(\dddot{\psi}^{n}, \ddot{\psi}^{n}, \dot{\psi}^{n}, \psi^{n} ; \ddot{\phi}^{a}, \dot{\phi}^{a}, \phi^{a} ; \dot{q}^{i}, q^{i}\right),
$$

where $n, a, i$ run from 1 to $\mathcal{N}, \mathcal{A}, \mathcal{I}$, respectively. In order to cover a wide class of Lagrangians up to the third-order time derivatives, we also include the variables $\phi^{a}$ that enter the Lagrangian up to their second-order time derivatives. We investigate the Hamiltonian analysis in section 3.1 to derive degeneracy conditions, and the Euler-Lagrange equations in section 3.2 to show the reduction to second-order system. For the special case $\mathcal{N}=1$ and $\mathcal{A}=0$, some part of degeneracy conditions are identically satisfied, for which 
we provide a brief explanation in appendix A. Instead of dealing with the Lagrangian (3.1), for the practical purpose, we consider an equivalent Lagrangian given by

$$
\begin{aligned}
L_{\mathrm{eq}} \equiv & L\left(\dot{Q}^{n}, Q^{n}, R^{n}, \psi^{n} ; \dot{Q}^{\mathcal{N}+a}, Q^{\mathcal{N}+a}, \phi^{a} ; \dot{q}^{i}, q^{i}\right) \\
& +\xi_{n}\left(\dot{\psi}^{n}-R^{n}\right)+\lambda_{n}\left(\dot{R}^{n}-Q^{n}\right)+\lambda_{\mathcal{N}+a}\left(\dot{\phi}^{a}-Q^{\mathcal{N}+a}\right),
\end{aligned}
$$

and denote $Q^{I}=\left(Q^{n}, Q^{\mathcal{N}+a}\right)$.

\subsection{Hamiltonian analysis}

The canonical momenta for $Q^{I}, q^{i}, R^{n}, \psi^{n}, \phi^{a}, \xi_{n}, \lambda_{I}$ are respectively given by

$$
P_{Q^{I}}=L_{I}, \quad p_{i}=L_{i}, \quad P_{R^{n}}=\lambda_{n}, \quad \pi_{\psi^{n}}=\xi_{n}, \quad \pi_{\phi^{a}}=\lambda_{\mathcal{N}+a}, \quad \rho_{\xi_{n}}=0, \quad \rho_{\lambda_{I}}=0,
$$

where $L_{I} \equiv \partial L / \partial \dot{Q}^{I}$ and $L_{i} \equiv \partial L / \partial \dot{q}^{i}$. Below we simply write $P_{Q^{I}} \rightarrow P_{I}$ when we denote all $I=(n, a)$ components, whereas we retain the notation $P_{Q^{n}}$ for $n$ components to distinguish it from $P_{R^{n}}$. The number of canonical variables are a priori $10 \mathcal{N}+6 \mathcal{A}+2 \mathcal{I}$.

From the latter six equations, we obtain $4 \mathcal{N}+2 \mathcal{A}$ primary constraints

$$
\begin{array}{lll}
\Phi_{n} \equiv P_{R^{n}}-\lambda_{n} \approx 0, & \Phi_{\mathcal{N}+a} \equiv \pi_{\phi^{a}}-\lambda_{\mathcal{N}+a} \approx 0, & \Phi_{\mathcal{N}+\mathcal{A}+n} \equiv \pi_{\psi^{n}}-\xi_{n} \approx 0 \\
\bar{\Phi}_{n} \equiv \rho_{\lambda_{n}} \approx 0, & \bar{\Phi}_{\mathcal{N}+a} \equiv \rho_{\lambda_{\mathcal{N}+a}} \approx 0, &
\end{array}
$$

At this moment, it is nontrivial whether the first two equations in (3.3) provide further constraints or not. However, if they do not provide constraints, the system has DOF more than the number of variables, and we end up with Ostrogradsky ghost. We thus assume the existence of an additional primary constraint in the following way. Let us consider the infinitesimal changes of $P_{I}, p_{i}$, which are related as

$$
\left(\begin{array}{c}
\delta P_{I}-L_{I x} \delta x \\
\delta p_{i}-L_{i x} \delta x
\end{array}\right)=K\left(\begin{array}{c}
\delta \dot{Q}^{J} \\
\delta \dot{q}^{j}
\end{array}\right),
$$

where the kinetic matrix $K$ is given by

$$
K \equiv\left(\begin{array}{cc}
L_{I J} & L_{I j} \\
L_{i J} & L_{i j}
\end{array}\right),
$$

and $x=\left(Q^{I}, R^{n}, \psi^{n}, \phi^{a}, q^{i}\right)$, and summation for overlapping $x$ is implicit. If $\operatorname{det} K \neq 0$, one can locally express $\dot{Q}^{I}, \dot{q}^{i}$ in terms of canonical variables, meaning that there is no further primary constraint. Therefore, we require $\operatorname{det} K=0$. More precisely, we require the maximal degeneracy of the part of $K$ corresponding to the higher derivatives to eliminate ghost DOFs. On the other hand, to avoid eliminating DOFs coming from $q^{i}$, we assume

$$
\operatorname{det} k \neq 0,
$$

where $k_{i j}$ is a sub-kinetic matrix defined by $k_{i j} \equiv L_{i j}$. Under this assumption, $K$ can be rewritten as

$$
K=R\left(\begin{array}{cc}
L_{I J}-L_{I i} k^{i j} L_{j J} & 0 \\
0 & k
\end{array}\right) S
$$


where $k^{i j}$ is the inverse matrix of $k_{i j}$ and

$$
R \equiv\left(\begin{array}{cc}
1 & A^{T} \\
0 & 1
\end{array}\right), \quad S \equiv\left(\begin{array}{cc}
1 & 0 \\
A & 1
\end{array}\right), \quad A_{I}^{i} \equiv k^{i j} L_{j I} .
$$

Now it is clear that the maximal degeneracy of the part of $K$ corresponding to the higher derivatives implies

$$
L_{I J}-L_{I i} L^{i j} L_{j J}=0,
$$

which is the first degeneracy condition we impose. Under this condition, (3.8) reads (see also appendix B.3 of [16])

$$
K=R\left(\begin{array}{ll}
0 & 0 \\
0 & k
\end{array}\right) S .
$$

Plugging (3.11) to (3.5) we obtain

$$
\begin{aligned}
& \delta P_{I}-L_{I i} L^{i j} \delta p_{j}=\left(L_{I x}-L_{I i} L^{i j} L_{j x}\right) \delta x, \\
& L^{i j} L_{I j} \delta \dot{Q}^{I}+\delta \dot{q}^{i}=L^{i j}\left(\delta p_{j}-L_{j x} \delta x\right) .
\end{aligned}
$$

We thus obtain additional primary constraints

$$
\Psi_{I} \equiv P_{I}-F_{I}\left(p_{i}, x\right) \approx 0,
$$

with

$$
F_{I p_{i}}=L^{i j} L_{I j}, \quad F_{I x}=L_{I x}-F_{I p_{i}} L_{i x} .
$$

The total Hamiltonian is given by

$$
\begin{aligned}
H_{T} & =H+\mu_{\alpha} \Phi_{\alpha}+\bar{\mu}_{\alpha} \bar{\Phi}_{\alpha}+\nu_{I} \Psi_{I} \\
H & =H_{0}+P_{R^{n}} Q^{n}+\pi_{\psi^{n}} R^{n}+\pi_{\phi^{a}} Q^{\mathcal{N}+a} \\
H_{0} & =\dot{Q}^{I} P_{I}+\dot{q}^{i} p_{i}-L\left(\dot{Q}^{n}, Q^{n}, R^{n}, \psi ; \dot{Q}^{\mathcal{N}+a}, Q^{\mathcal{N}+a}, \phi^{a} ; \dot{q}^{i}, q^{i}\right),
\end{aligned}
$$

where $\Phi_{\alpha}=\left(\Phi_{n}, \Phi_{\mathcal{N}+a}, \Phi_{\mathcal{N}+\mathcal{A}+n}\right)$ with $\alpha=1, \cdots, 2 \mathcal{N}+\mathcal{A}$ and so does $\bar{\Phi}_{\alpha}$, and $\mu_{\alpha}, \bar{\mu}_{\alpha}, \nu_{I}$ are the Lagrange multipliers associated with the primary constraints $\Phi_{\alpha}, \bar{\Phi}_{\alpha}, \Psi_{I}$, respectively. The momenta $P_{R^{n}}, \pi_{\psi^{n}}, \pi_{\phi^{a}}$ show up in the Hamiltonian only through the linear terms, which lead to the Ostrogradsky instability. We shall see that the secondary constraints fix $P_{R^{n}}, \pi_{\phi^{a}}$, and the tertiary constraints fix $\pi_{\psi^{n}}$.

To guarantee that the primary constraints $\Phi_{\alpha}, \bar{\Phi}_{\alpha}, \Psi_{I}$ are satisfied through time evolution, the consistency conditions $\dot{\Phi}_{\alpha} \approx 0, \dot{\bar{\Phi}}_{\alpha} \approx 0, \dot{\Psi}_{I} \approx 0$ should be satisfied. From $\dot{\Phi}_{\alpha} \approx 0$, we obtain equations for $\bar{\mu}_{\alpha}$ as

$$
\bar{\mu}_{\alpha} \approx\left\{\Phi_{\alpha}, H\right\}+\nu_{I}\left\{\Phi_{\alpha}, \Psi_{I}\right\}
$$

which read

$$
\begin{aligned}
\bar{\mu}_{n} & \approx-\pi_{\psi^{n}}+L_{R^{n}}+\nu_{I}\left\{\Phi_{n}, \Psi_{I}\right\}, \\
\bar{\mu}_{\mathcal{N}+a} & \approx L_{\phi^{a}}+\nu_{I}\left\{\Phi_{\mathcal{N}+a}, \Psi_{I}\right\}, \\
\bar{\mu}_{\mathcal{N}+\mathcal{A}+n} & \approx L_{\psi^{n}}+\nu_{I}\left\{\Phi_{\mathcal{N}+\mathcal{A}+n}, \Psi_{I}\right\} .
\end{aligned}
$$


On the other hand, $\dot{\bar{\Phi}}_{\alpha} \approx 0$ fixes $\mu_{\alpha}$ as

$$
\mu_{\alpha} \approx 0 .
$$

Therefore the consistency conditions for the primary constraints $\Phi_{\alpha}, \bar{\Phi}_{\alpha}$ determine Lagrange multipliers $\bar{\mu}_{\alpha}, \mu_{\alpha}$, respectively, and do not generate secondary constraints. The remaining consistency conditions for the primary constraints $\Psi_{I}$ are

$$
0 \approx \dot{\Psi}_{I}=\left\{\Psi_{I}, H\right\}+\nu_{J}\left\{\Psi_{I}, \Psi_{J}\right\},
$$

where we substituted (3.18). As shown in [14], the appearance of the matrix $\left\{\Psi_{I}, \Psi_{J}\right\}$ is the nature of the multi-variable system, and if $\left\{\Psi_{I}, \Psi_{J}\right\}$ is nondegenerate, this system suffers from ghost DOFs. We thus need further constraints to eliminate them. To make all the equations give secondary constraints, we impose the second degeneracy conditions

$$
\left\{\Psi_{I}, \Psi_{J}\right\}=F_{J Q^{I}}-F_{I Q^{J}}+F_{I q^{i}} F_{J p_{i}}-F_{I p_{i}} F_{J q^{i}}=0 .
$$

Under the second degeneracy conditions (3.20) we obtain secondary constraints

$$
\Upsilon_{I} \equiv-\left\{\Psi_{I}, H\right\} \approx 0
$$

which read

$$
\begin{aligned}
\Upsilon_{n} & =P_{R^{n}}-L_{Q^{n}}+F_{n p_{i}} L_{q^{i}}+\dot{x} F_{n x} \equiv P_{R^{n}}-G_{n}, \\
\Upsilon_{\mathcal{N}+a} & =\pi_{\phi^{a}}-L_{Q^{\mathcal{N}+a}}+F_{\mathcal{N}+a, p_{i}} L_{q^{i}}+\dot{x} F_{\mathcal{N}+a, x} \equiv \pi_{\phi^{a}}-G_{\mathcal{N}+a},
\end{aligned}
$$

which fix $P_{R^{n}}, \pi_{\phi^{a}}$, eliminating Ostrogradsky instability coming from terms linear in them in the Hamiltonian (3.15).

Note that, for the case $\mathcal{N}=1$ and $\mathcal{A}=0$, the Poisson bracket is $\left\{\Psi_{I}, \Psi_{J}\right\} \rightarrow\{\Psi, \Psi\}$ which identically vanishes. Hence, as mentioned earlier, the degeneracy conditions corresponding to (3.20) are identically satisfied, and one obtains the secondary constraints corresponding to (3.22) automatically.

We can show that $G_{I}=G_{I}\left(p_{i}, x\right)$ as follows. By using the second equation of (3.12), we can show that $\delta \dot{Q}^{I}$ and $\delta \dot{q}^{i}$ terms of the variation of $L_{x}-X_{I p_{i}} L_{q^{i}}+\dot{y} X_{I y}$ for general $X_{I}=X_{I}\left(p_{i}, x\right)$ can be given by

$$
\delta\left(L_{x}-X_{I p_{i}} L_{q^{i}}-\dot{y} X_{I y}\right) \supset\left(F_{J x}-X_{I Q^{J}}+X_{I q^{i}} F_{J p_{i}}-X_{I p_{i}} F_{J q^{i}}\right) \delta \dot{Q}^{J} .
$$

Applying this relation to $G_{I}=L_{Q^{I}}-F_{I p_{i}} L_{q^{i}}-\dot{x} F_{I x}$, we obtain

$$
\delta G_{I} \supset\left(F_{J Q^{I}}-F_{I Q^{J}}+F_{J p_{i}} F_{I q^{i}}-F_{I p_{i}} F_{J q^{i}}\right) \delta \dot{Q}^{J} .
$$

We see that the coefficient precisely coincides with the second degeneracy conditions (3.20). We thus conclude $G_{I}=G_{I}\left(p_{i}, x\right)$. For the case $\mathcal{N}=1, \mathcal{A}=0$, one can show the right hand side of (3.24) identically vanishes.

The consistency conditions for the secondary constraints are given by

$$
0 \approx \dot{\Upsilon}_{I}=\left\{\Upsilon_{I}, H\right\}+\nu_{J}\left\{\Upsilon_{I}, \Psi_{J}\right\}
$$


As mentioned earlier, among $\Upsilon_{I}=\left(\Upsilon_{n}, \Upsilon_{\mathcal{N}+a}\right)$, the latter part are constraints eliminating Ostrogradsky ghost associated with $\pi_{\phi^{a}}$. We thus would like to stop the reduction of $\phi$ sector, while we still need further constraints to eliminate Ostrogradsky ghost in $\psi$ sector. Hence, we require $\operatorname{det}\left\{\Upsilon_{\mathcal{N}+a}, \Psi_{\mathcal{N}+b}\right\} \neq 0$ by which $\nu_{\mathcal{N}+a}$ are fixed. To remove ghost DOFs from $\psi$ sector under the condition $\operatorname{det}\left\{\Upsilon_{\mathcal{N}+a}, \Psi_{\mathcal{N}+b}\right\} \neq 0$, one may be tempted to impose the third degeneracy conditions as

$$
\left\{\Upsilon_{n}, \Psi_{m}\right\}-\left\{\Upsilon_{n}, \Psi_{\mathcal{N}+a}\right\}\left\{\Upsilon_{\mathcal{N}+b}, \Psi_{\mathcal{N}+a}\right\}^{-1}\left\{\Upsilon_{\mathcal{N}+b}, \Psi_{m}\right\}=0
$$

so that $\left\{\Upsilon_{I}, \Psi_{J}\right\}$ can be decomposed as

$$
\left\{\Upsilon_{I}, \Psi_{J}\right\}=R^{\prime}\left(\begin{array}{lc}
0 & 0 \\
0 & \left\{\Upsilon_{\mathcal{N}+a}, \Psi_{\mathcal{N}+b}\right\}
\end{array}\right) S^{\prime},
$$

with some nontrivial $R^{\prime}, S^{\prime}$, in parallel to (3.10) and (3.11). This time, for simplicity, we impose

$$
\left\{\Upsilon_{n}, \Psi_{I}\right\}=F_{I R^{n}}-G_{n Q^{I}}+G_{n q^{i}} F_{I p_{i}}-G_{n p_{i}} F_{I q^{i}}=0,
$$

as the third degeneracy conditions to ensure the structure

$$
\left\{\Upsilon_{I}, \Psi_{J}\right\}=\left(\begin{array}{cc}
0 & 0 \\
\left\{\Upsilon_{\mathcal{N}+a}, \Psi_{m}\right\} & \left\{\Upsilon_{\mathcal{N}+a}, \Psi_{\mathcal{N}+b}\right\}
\end{array}\right),
$$

where $\operatorname{det}\left\{\Upsilon_{\mathcal{N}+a}, \Psi_{\mathcal{N}+b}\right\} \neq 0$. Plugging (3.29) into (3.25), the first row yields the tertiary constraints given by

$$
0 \approx \Lambda_{n} \equiv-\left\{\Upsilon_{n}, H\right\}=\pi_{\psi^{n}}-L_{R^{n}}+G_{n p_{i}} L_{q^{i}}+\dot{x} G_{n x} \equiv \pi_{\psi^{n}}-I_{n}\left(p_{i}, x\right),
$$

where we have again used (3.23) to show that $I_{n}=I_{n}\left(p_{i}, x\right)$. Thus, the tertiary constraints fix $\pi_{\psi^{n}}$. On the other hand, the remaining $\mathcal{A}$ components of (3.25) give $\mathcal{A}$ equations for

$$
\left\{\Upsilon_{\mathcal{N}+a}, \Psi_{J}\right\} \nu_{J} \approx-L_{\phi^{a}}+G_{\mathcal{N}+a, p_{i}} L_{q^{i}}+\dot{x} G_{\mathcal{N}+a, x}
$$

Since $\operatorname{det}\left\{\Upsilon_{\mathcal{N}+a}, \Psi_{\mathcal{N}+b}\right\} \neq 0$, this equation fix $\nu_{\mathcal{N}+a}$ as expected. We shall see in (3.57) that the right hand side is vanishing by virtue of $\mathrm{EOM}$ for $\phi^{a}$. For the case $\mathcal{N}=1, \mathcal{A}=0$, the Poisson bracket is $\left\{\Upsilon_{I}, \Psi_{J}\right\} \rightarrow\{\Upsilon, \Psi\}$ and one simply needs to impose $\{\Upsilon, \Psi\}=0$ as the degeneracy condition.

Therefore, we have fixed all the linear momentum terms $P_{R^{n}}, \pi_{\psi^{n}}, \pi_{\phi^{a}}$ in the Hamiltonian (3.15). However, as demonstrated in [21] for the quadratic model, the salient feature that the Ostrogradsky ghosts are not completely eliminated even after all the linear terms in momenta have been removed by the constraints is expected to be generic in the higher derivative theories with more than second time-derivatives in the Lagrangian. This is because the canonical variables $Q^{n}$ correspond to the second time derivatives of $\psi^{n}$ and could become the source of the Ostrogradsky ghosts.

In the present case with general Lagrangian, an explicit redefinition of variables that reveals the hidden ghost is not trivial. Instead, we use the counting of the number of phase 
space variables. All the phase space variables of the current system (3.2) are

$$
\begin{array}{cccccccc}
Q^{I} & q^{i} & R^{n} & \psi^{n} & \phi^{a} & \xi_{n} & \lambda_{I} \\
\hline P_{Q^{I}} & p_{i} & P_{R^{n}} & \pi_{\psi^{n}} & \pi_{\phi^{a}} & \rho_{\xi_{n}} & \rho_{\lambda_{I}}
\end{array},
$$

where the boxed variables are fixed in terms of other variables via constraints obtained so far. Therefore, we currently have $3 \mathcal{N}+2 \mathcal{A}+2 \mathcal{I}$ free variables in phase space. The original Lagrangian (3.1) depends on $\psi^{n}, \phi^{a}, q^{i}$ and we would like to have a theory such that these variables behave as if they are "ordinary" variables corresponding to $2(\mathcal{N}+\mathcal{A}+\mathcal{I})$ free variables in phase space. Therefore, from (3.32) the current system has $\mathcal{N}$ extra phase space variables, and we assume that they are the hidden Ostrogradsky ghosts, which do not appear in the Hamiltonian as linear momentum terms. Generalizing the result obtained in [21], we expect that for some simple cases it is possible to find out an explicit redefinition of variables to reveal the hidden ghost as a term linear in $Q_{n}$ in the Hamiltonian.

Based on these considerations, to eliminate the hidden Ostrogradsky ghosts, we require that the consistency conditions for the tertiary constraints (3.30)

$$
0 \approx \dot{\Lambda}_{n}=\left\{\Lambda_{n}, H_{T}\right\}=\left\{\Lambda_{n}, H\right\}+\nu_{J}\left\{\Lambda_{n}, \Psi_{J}\right\},
$$

does not determine any Lagrange multipliers, and hence generate the quaternary constraints. Along the same line as the third degeneracy condition (3.28), as the simplest case, although not the most general, we require

$$
\left\{\Lambda_{n}, \Psi_{I}\right\}=F_{I \psi^{n}}-I_{n Q^{I}}+I_{n q^{i}} F_{I p_{i}}-I_{n p_{i}} F_{I q^{i}}=0,
$$

as the fourth degeneracy conditions. Then, the consistency conditions (3.33) for $\Lambda_{n}$ yield the following quaternary constraints,

$$
0 \approx \Omega_{n} \equiv-\left\{\Lambda_{n}, H\right\}=-L_{\psi^{n}}+I_{n p_{i}} L_{q^{i}}+\dot{x} I_{n x}
$$

which fix the $\mathcal{N}$ phase space variables, precisely matching the number of $Q_{n}$, as expected. Again, using (3.23) we can show that $\Omega_{n}=-J_{n}\left(p_{i}, x\right)$.

For the case $\mathcal{N}=1, \mathcal{A}=0$, one can show $\{\Lambda, \Psi\}=0$ identically holds (see appendix A for the proof), and the quaternary constraint is automatically obtained. This makes sense since the absence of such constraint would lead to the equations of motion containing third time-derivative of a single variable only, which is incompatible with the nature of Euler-Lagrange equations.

The consistency conditions for $\Omega_{n}$ yield

$$
\left\{\Omega_{n}, \Psi_{I}\right\} \nu_{I} \approx J_{n p_{i}} L_{q^{i}}+\dot{x} J_{n x},
$$

whose right hand side shall be shown to be vanishing in (3.59) by virtue of time derivative of EOM for $\psi^{n}$. Thus, (3.31) and (3.36) form a system of $\mathcal{N}+\mathcal{A}$ equations for $\nu_{I}$. Since we have reduced the number of the unconstrained canonical variables to $2(\mathcal{N}+\mathcal{A}+\mathcal{I})$, we do not impose further constraints. In other words, we require all the Lagrange multipliers $\nu_{I}$ are determined by (3.31) and (3.36). Denoting

$$
\hat{\Omega}_{I} \equiv\left(\Omega_{n}, \Upsilon_{\mathcal{N}+a}\right),
$$


and

$$
Z_{I J} \equiv\left\{\hat{\Omega}_{I}, \Psi_{J}\right\}
$$

we require each submatrix of $Z_{I J}$ is nondegenerate:

$$
\operatorname{det} Z_{a b} \neq 0, \quad \operatorname{det} Z_{n m} \neq 0 .
$$

Under this condition we obtain

$$
\nu_{I} \approx 0 .
$$

The number of constraints is

$$
\begin{aligned}
\Phi_{\alpha}, \bar{\Phi}_{\alpha} & : 4 \mathcal{N}+2 \mathcal{A}, \\
\Psi_{I} & : \mathcal{N}+\mathcal{A}, \\
\hat{\Omega}_{I} & : \mathcal{N}+\mathcal{A}, \\
\Upsilon_{n} & : \mathcal{N} \\
\Lambda_{n} & : \mathcal{N},
\end{aligned}
$$

and the total number is thus $8 \mathcal{N}+4 \mathcal{A}$. Using the definition $\Upsilon_{I}=-\left\{\Psi_{I}, H\right\}$ and the Jacobi identity we can show

$$
\begin{aligned}
\left\{\Upsilon_{I}, \Upsilon_{n}\right\} & =\left\{\Lambda_{n}, \Psi_{I}\right\}+\left\{\left\{\Upsilon_{n}, \Psi_{I}\right\}, H\right\}=0, \\
\left\{\Upsilon_{I}, \Lambda_{n}\right\} & =\left\{\Omega_{n}, \Psi_{I}\right\}+\left\{\left\{\Lambda_{n}, \Psi_{I}\right\}, H\right\}=Z_{n I}, \\
Z_{a n} & =\left\{\Upsilon_{\mathcal{N}+a}, \Psi_{n}\right\}=\left\{\Upsilon_{n}, \Psi_{\mathcal{N}+a}\right\}=0 .
\end{aligned}
$$

With this in mind, the Dirac matrix is given by

\begin{tabular}{c|cccccc} 
& $\Phi_{\beta}$ & $\bar{\Phi}_{\beta}$ & $\Psi_{J}$ & $\hat{\Omega}_{J}$ & $\Upsilon_{m}$ & $\Lambda_{m}$ \\
\hline$\Phi_{\alpha}$ & 0 & -1 & $*$ & $*$ & $*$ & $*$ \\
$\bar{\Phi}_{\alpha}$ & 1 & 0 & 0 & 0 & 0 & 0 \\
$\Psi_{I}$ & $*$ & 0 & 0 & $-Z_{J I}$ & 0 & 0 \\
$\hat{\Omega}_{I}$ & $*$ & 0 & $Z_{I J}$ & $*$ & $*$ & $*$ \\
$\Upsilon_{n}$ & $*$ & 0 & 0 & $*$ & 0 & $Z_{m n}$ \\
$\Lambda_{n}$ & $*$ & 0 & 0 & $*$ & $-Z_{n m}$ & $*$
\end{tabular}

and the determinant of the Dirac matrix is given by

$$
\left(\operatorname{det} Z_{a b}\right)^{2}\left(\operatorname{det} Z_{n m}\right)^{4}
$$

which does not vanish by virtue of (3.39). Therefore, since all the $8 \mathcal{N}+4 \mathcal{A}$ constraints are second class, the number of DOF is

$$
\frac{1}{2}[10 \mathcal{N}+6 \mathcal{A}+2 \mathcal{I}-(8 \mathcal{N}+4 \mathcal{A})]=\mathcal{N}+\mathcal{A}+\mathcal{I} .
$$




\section{$3.2 \quad$ Euler-Lagrange equation}

The Euler-Lagrange equation for (3.2) is given by

$$
\begin{aligned}
\dot{L}_{i}-L_{q^{i}} & =0, \\
\dot{L}_{I}-L_{Q^{I}}+\lambda_{I} & =0, \\
L_{R^{n}}-\xi_{n}-\dot{\lambda}_{n} & =0, \\
L_{\psi^{n}}-\dot{\xi}_{n} & =0, \\
L_{\phi^{a}}-\dot{\lambda}_{N+a} & =0, \\
Q^{n}-\dot{R}^{n} & =0, \\
R^{n}-\dot{\psi}^{n} & =0, \\
Q^{N+a}-\dot{\phi}^{a} & =0 .
\end{aligned}
$$

To obtain EOM for $\psi^{n}, \phi^{a}, q^{i}$ we successively take time derivative of the Lagrange multipliers $\lambda_{I}, \xi_{n}$.

First, we begin with $\lambda_{I}$. From (3.47), a priori $\lambda_{I}$ depends on $\ddot{Q}^{I}$ which we would like to avoid. Using the first degeneracy condition (3.10) or the additional primary constraints (3.13), $L_{I}=F_{I}\left(L_{i}, x\right)$ with the relations (3.14), we can show (3.46) and (3.47) can be transformed as

$$
\begin{aligned}
\lambda_{I} & =L_{Q^{I}}-F_{I p_{i}} L_{q^{i}}-\dot{x} F_{I x}, \\
\mathcal{E}_{i} & \equiv \ddot{q}^{i}+F_{I p_{i}} \ddot{Q}^{I}-L^{i j}\left(L_{q^{j}}-\dot{x} L_{j x}\right)=0 .
\end{aligned}
$$

The first equation (3.54) corresponds to the secondary constraints (3.22).

Second, we take time derivative of (3.54) to obtain $\xi_{n}$ from (3.48), and EOM for $\phi^{a}$ from (3.50). Again, to avoid for them to depend on $\ddot{Q}^{I}$, we impose $\lambda_{I}=G_{I}\left(L_{i}, x\right)$. Indeed, in (3.24) we showed it holds by virtue of the second degeneracy condition (3.20). Thus $\xi_{n}$ and EOM for $\phi^{a}$ does not depend on $\ddot{Q}^{I}$. In fact, from (3.48) we obtain

$$
\xi_{n}=L_{R^{n}}-G_{n p_{i}} L_{q^{i}}-\dot{x} G_{n x}
$$

which corresponds to the tertiary constraints (3.30). Also, from (3.50) we obtain EOM for $\phi^{a}$

$$
\mathcal{E}_{a} \equiv L_{\phi^{a}}-G_{\mathcal{N}+a, p_{i}} L_{q^{i}}-\dot{x} G_{\mathcal{N}+a, x}=0
$$

which corresponds to the right hand side of (3.31).

Third, we take time derivative of (3.56) to obtain EOM for $\psi^{n}$ from (3.49). Again, to avoid its $\ddot{Q}^{I}$ dependency, we impose $\xi_{n}=I_{n}\left(L_{i}, x\right)$, which has been actually shown in the previous subsection by using the third degeneracy condition (3.28). From (3.49) we obtain EOM for $\psi^{n}$

$$
\mathcal{E}_{n} \equiv L_{\psi^{n}}-I_{n p_{i}} L_{q^{i}}-\dot{x} I_{n x}=0,
$$

which corresponds to the quaternary constraints (3.35).

We thus obtain EOM for $q^{i}, \phi^{a}, \psi^{n}$ as (3.55), (3.57), (3.58), but they still contain higher derivatives. Below we construct a set of EOMs with derivatives up to second-order. 
We derive another independent EOM by taking time derivative of (3.58). To avoid its $\ddot{Q}^{I}$ dependency, we impose $\mathcal{E}_{n}=J_{n}\left(L_{i}, x\right)$ which holds by virtue of the fourth degeneracy condition (3.34). Therefore,

$$
0=\dot{J}_{n}=J_{n p_{i}} L_{q^{i}}+\dot{x} J_{n x}
$$

which coincides with the right hand side of (3.36). Generalizing the derivation of eq. (24) from eq. (23) in [21], we expect that in general the condition (3.39) guarantees that we can solve (3.57)-(3.59) for $\dot{Q}^{n}, Q^{n}, \dot{Q}_{\mathcal{N}+a}$ and obtain

$$
\begin{aligned}
\dot{Q}^{n} & =\dot{Q}^{n}\left(\dot{q}^{i}, Q^{\mathcal{N}+a}, R^{n}, \psi^{n}, \phi^{a}, q^{i}\right), \\
Q^{n} & =Q^{n}\left(\dot{q}^{i}, Q^{\mathcal{N}+a}, R^{n}, \psi^{n}, \phi^{a}, q^{i}\right), \\
\dot{Q}^{\mathcal{N}+a} & =\dot{Q}^{\mathcal{N}+a}\left(\dot{q}^{i}, Q^{\mathcal{N}+a}, R^{n}, \psi^{n}, \phi^{a}, q^{i}\right) .
\end{aligned}
$$

The equations (3.61), (3.62) are EOMs containing at most $\ddot{\psi}^{n}=Q^{n}, \ddot{\phi}^{a}=\dot{Q}^{\mathcal{N}+a}$, respectively. Taking time derivative of (3.60) and (3.62), and using these equations we obtain

$$
\ddot{Q}^{I}=\ddot{Q}^{I}\left(\ddot{q}^{i}, \dot{q}^{i}, Q^{\mathcal{N}+a}, R^{n}, \psi^{n}, \phi^{a}, q^{i}\right)
$$

By substituting (3.60)-(3.63) to (3.55), we obtain EOM containing at most $\ddot{q}^{i}$. Combining it with (3.61), (3.62), we thus obtain a system of $\mathcal{N}+\mathcal{A}+\mathcal{I}$ EOMs that contain at most $\ddot{\psi}^{n}, \ddot{\phi}^{a}, \ddot{q}^{i}$.

\section{Lagrangian with arbitrary higher-order derivatives}

Finally we extend the analyses in section 3 for the Lagrangian with third-order derivatives to that with arbitrary higher order derivatives. We explore the following Lagrangian involving arbitrary higher $(d+1)$-th order derivatives:

$$
L=L\left(\phi^{i_{0}}, \dot{\phi}^{i_{0}} ; \phi^{i_{1}}, \dot{\phi}^{i_{1}}, \ddot{\phi}^{i_{1}} ; \phi^{i_{2}}, \dot{\phi}^{i_{2}}, \ddot{\phi}^{i_{2}}, \dddot{\phi}^{i_{2}} ; \cdots ; \phi^{i_{d}}, \dot{\phi}^{i_{d}}, \cdots, \phi^{i_{d}(d+1)}\right) .
$$

Here, the index $i_{k}$ counts the number of $\phi(t)$ variables and runs

$$
\begin{aligned}
i_{0} & =1, \cdots, n_{0}, \\
i_{1} & =n_{0}+1, \cdots, n_{0}+n_{1}, \\
& \vdots \\
i_{d} & =\sum_{k=0}^{d-1} n_{k}+1, \cdots, \sum_{k=0}^{d} n_{k},
\end{aligned}
$$

and $\phi^{i_{k}}(t)$ receives $(k+1)$-th order derivative. Note that the numbering and the order of time derivative are off by 1 for later convenience. We introduce the notation

$$
Q_{00}^{i_{0}} \equiv \phi^{i_{0}}, \quad Q_{10}^{i_{1}} \equiv \phi^{i_{1}}, \quad \cdots \quad Q_{d 0}^{i_{d}} \equiv \phi^{i_{d}}
$$


and the auxiliary variables to rewrite the Lagrangian as

$$
\begin{aligned}
L_{\mathrm{eq}}= & L\left(Q_{00}^{i_{0}}, \dot{Q}_{00}^{i_{0}} ; Q_{10}^{i_{1}}, Q_{11}^{i_{1}}, \dot{Q}_{11}^{i_{1}} ; Q_{20}^{i_{2}}, Q_{21}^{i_{2}}, Q_{22}^{i_{2}}, \dot{Q}_{22}^{i_{2}} ; \cdots ; Q_{d 0}^{i_{d}}, Q_{d 1}^{i_{d}}, \cdots, Q_{d d}^{i_{d}}, \dot{Q}_{d d}^{i_{d}}\right) \\
& +\lambda_{10}^{i_{1}}\left(\dot{Q}_{10}^{i_{1}}-Q_{11}^{i_{1}}\right) \\
& +\lambda_{20}^{i_{2}}\left(\dot{Q}_{20}^{i_{2}}-Q_{21}^{i_{2}}\right)+\lambda_{21}^{i_{2}}\left(\dot{Q}_{21}^{i_{2}}-Q_{22}^{i_{2}}\right) \\
& +\cdots \\
& +\lambda_{d 0}^{i_{d}}\left(\dot{Q}_{d 0}^{i_{d}}-Q_{d 1}^{i_{d}}\right)+\lambda_{d 1}^{i_{d}}\left(\dot{Q}_{d 1}^{i_{d}}-Q_{d 2}^{i_{d}}\right)+\cdots+\lambda_{d, d-1}^{i_{d}}\left(\dot{Q}_{d, d-1}^{i_{d}}-Q_{d d}^{i_{d}}\right) .
\end{aligned}
$$

Therefore, we have $\{Q, \lambda\}$ and their canonical momenta $\{P, \rho\}$ which we classify as

$$
\begin{aligned}
& q^{i} \equiv\left(Q_{00}^{i_{0}}\right), \quad p_{i} \equiv\left(P_{00}^{i_{0}}\right), \\
& \tilde{Q}_{I_{1}}^{(0)} \equiv\left(Q_{11}^{i_{1}}, Q_{22}^{i_{2}}, \cdots, Q_{d d}^{i_{d}}\right), \quad \tilde{P}_{I_{1}}^{(0)} \equiv\left(P_{11}^{i_{1}}, P_{22}^{i_{2}}, \cdots, P_{d d}^{i_{d}}\right), \\
& \boldsymbol{Q} \equiv\left(\begin{array}{cccc}
Q_{10}^{i_{1}} & & & \\
Q_{20}^{i_{2}} & Q_{21}^{i_{2}} & & \\
\vdots & & \ddots & \\
Q_{d 0}^{i_{d}} & Q_{d 1}^{i_{d}} & \cdots & Q_{d, d-1}^{i_{d}}
\end{array}\right), \quad \boldsymbol{P} \equiv\left(\begin{array}{cccc}
P_{10}^{i_{1}} & & & \\
P_{20}^{i_{2}} & P_{21}^{i_{2}} & & \\
\vdots & & \ddots & \\
P_{d 0}^{i_{d}} & P_{d 1}^{i_{d}} & \cdots & P_{d, d-1}^{i_{d}}
\end{array}\right), \\
& \boldsymbol{\lambda} \equiv\left(\begin{array}{cccc}
\lambda_{10}^{i_{1}} & & & \\
\lambda_{20}^{i_{2}} & \lambda_{21}^{i_{2}} & & \\
\vdots & & \ddots & \\
\lambda_{d 0}^{i_{d}} & \lambda_{d 1}^{i_{d}} & \cdots & \lambda_{d, d-1}^{i_{d}}
\end{array}\right), \quad \boldsymbol{\rho} \equiv\left(\begin{array}{cccc}
\rho_{10}^{i_{1}} & & & \\
\rho_{20}^{i_{2}} & \rho_{21}^{i_{2}} & & \\
\vdots & & \ddots & \\
\rho_{d 0}^{i_{d}} & \rho_{d 1}^{i_{d}} & \cdots & \rho_{d, d-1}^{i_{d}}
\end{array}\right),
\end{aligned}
$$

where $I_{1}=\left(i_{1}, i_{2}, \cdots, i_{d}\right)$. The total number of the canonical variables is thus a priori

$$
\begin{aligned}
N_{\text {can }} & =2 \sum_{k=0}^{d}(k+1) n_{k}+2 \sum_{k=1}^{d} k n_{k} \\
& =2 \sum_{k=0}^{d} n_{k}+4 \sum_{k=1}^{d} k n_{k} .
\end{aligned}
$$

Below we consider how to remove $4 \sum_{k=1}^{d} k n_{k}$ by constraints.

\subsection{Hamiltonian analysis}

The canonical momenta are defined as

$$
p_{i}=L_{i}, \quad \tilde{P}_{I_{1}}^{(0)}=L_{I_{1}}, \quad \boldsymbol{P}=\boldsymbol{\lambda}, \quad \boldsymbol{\rho}=\mathbf{0},
$$

where $L_{I_{1}} \equiv \partial L / \partial \dot{\tilde{Q}}_{I_{1}}^{(0)}$. First, from the latter two equations we obtain the primary constraints

$$
\Phi \equiv P-\lambda \approx 0, \quad \bar{\Phi} \equiv \rho \approx 0 .
$$

As we shall see, they are second class constraints and thus constrain only $\boldsymbol{\lambda}$ and $\boldsymbol{\rho}$. Next we focus on the former two equations. The $q^{i}$ and $Q^{I}$ sectors are parallel to those in the previous section. Thus we assume $\operatorname{det} L_{i j} \neq 0$, and impose the first degeneracy condition

$$
L_{I_{1} J_{1}}-L_{I_{1} i} L^{i j} L_{j J_{1}}=0 \text {, }
$$


which is equivalent to the additional primary constraints

$$
\tilde{\Psi}_{I_{1}}^{(0)} \equiv \tilde{P}_{I_{1}}^{(0)}-\tilde{F}_{I_{1}}^{(0)}\left(p_{i}, x\right) \approx 0,
$$

where

$$
\tilde{\Psi}_{I_{1}}^{(0)}=\left(\Psi_{11}^{i_{1}}, \Psi_{22}^{i_{2}}, \cdots, \Psi_{d d}^{i_{d}}\right)
$$

and $x=\left(q^{i}, \tilde{Q}_{I_{1}}^{(0)}, \boldsymbol{Q}\right)$.

To write down the total Hamiltonian in a simpler form we introduce the notation in addition to $\tilde{Q}_{I_{1}}^{(0)} \equiv\left(Q_{11}^{i_{1}}, Q_{22}^{i_{2}}, \cdots, Q_{d d}^{i_{d}}\right)$

$$
\begin{aligned}
\tilde{Q}_{I_{1}}^{(1)} & \equiv\left(Q_{10}^{i_{1}}, Q_{21}^{i_{2}}, Q_{32}^{i_{3}}, \cdots, Q_{d, d-1}^{i_{d}}\right), \\
\tilde{Q}_{I_{2}}^{(2)} & \equiv\left(Q_{20}^{i_{2}}, Q_{31}^{i_{3}}, \cdots, Q_{d, d-2}^{i_{d}}\right), \\
& \vdots \\
\tilde{Q}_{I_{d-1}}^{(d-1)} & \equiv\left(Q_{d-1,0}^{i_{d-1}}, Q_{d 1}^{i_{d}}\right), \\
\tilde{Q}_{I_{d}}^{(d)} & \equiv\left(Q_{d 0}^{i_{d}}\right)
\end{aligned}
$$

which decompose the matrix $\boldsymbol{Q}$ into $d$ vectors, picking up the arguments from left top to right down. Here $I_{k}=\left(i_{k}, \cdots, i_{d}\right)$, and thus we can decompose

$$
\tilde{Q}_{I_{k}}^{(k)}=\left(Q_{k 0}^{i_{k}}, \tilde{Q}_{I_{k+1}}^{(k)}\right), \quad \tilde{Q}_{I_{k+1}}^{(k)}=\left(Q_{k+1,1}^{i_{k+1}}, Q_{k+2,2}^{i_{k+2}}, \cdots, Q_{d, d-k}^{i_{d}}\right),
$$

which we exploit below to isolate the first argument. We also define $\tilde{P}_{I_{k}}^{(k)}$ in the same way:

$$
\tilde{P}_{I_{k}}^{(k)} \equiv\left(P_{k 0}^{i_{k}}, P_{k+1,1}^{i_{k+1}}, \cdots, P_{d, d-k}^{i_{d}}\right) .
$$

With this notation, the Lagrangian (4.4) simplifies as

$$
L_{\mathrm{eq}}=L\left(q^{i}, \dot{q}^{i} ; \tilde{Q}_{I_{1}}^{(0)}, \dot{\tilde{Q}}_{I_{1}}^{(0)} ; \tilde{Q}_{I_{1}}^{(1)}, \tilde{Q}_{I_{2}}^{(2)}, \cdots, \tilde{Q}_{I_{d}}^{(d)}\right)+\sum_{k=1}^{d} \tilde{\lambda}_{I_{k}}^{(k)}\left(\dot{\tilde{Q}}_{I_{k}}^{(k)}-\tilde{Q}_{I_{k}}^{(k-1)}\right) .
$$

The total Hamiltonian is then given by

$$
\begin{aligned}
H_{T} & =H+\mu_{\alpha} \Phi_{\alpha}+\bar{\mu}_{\alpha} \bar{\Phi}_{\alpha}+\tilde{\nu}_{I_{1}}^{(0)} \tilde{\Psi}_{I_{1}}^{(0)}, \\
H & =H_{0}+\tilde{Q}_{I_{1}}^{(0)} \tilde{P}_{I_{1}}^{(1)}+\tilde{Q}_{I_{2}}^{(1)} \tilde{P}_{I_{2}}^{(2)}+\cdots+\tilde{Q}_{I_{d}}^{(d-1)} \tilde{P}_{I_{d}}^{(d)}, \\
H_{0} & =\dot{\tilde{Q}}_{I_{1}}^{(0)} \tilde{P}_{I_{1}}^{(0)}+\dot{q}^{i} p_{i}-L,
\end{aligned}
$$

where $\Phi_{\alpha}=\left(\Phi_{10}^{i_{1}}, \Phi_{20}^{i_{2}}, \Phi_{21}^{i_{2}}, \cdots, \Phi_{d 0}^{i_{d}}, \cdots, \Phi_{d, d-1}^{i_{d}}\right)$ denotes the $\sum_{k=1}^{d} k n_{k}$ constraints, and so does $\bar{\Phi}_{\alpha}$. Clearly, the linear terms $\tilde{Q}_{I_{k}}^{(k-1)} \tilde{P}_{I_{k}}^{(k)}$ cause Ostrogradsky instabilities. Below we show how to remove them by imposing constraints to $\tilde{P}_{I_{k}}^{(k)}$.

The consistency conditions $\dot{\bar{\Phi}}_{\alpha} \approx 0$ and $\dot{\Phi}_{\alpha} \approx 0$ respectively give

$$
\mu_{\alpha} \approx 0, \quad \bar{\mu}_{\alpha} \approx\left\{\Phi_{\alpha}, H\right\}+\tilde{\nu}_{I_{1}}^{(0)}\left\{\Phi_{\alpha}, \tilde{\Psi}_{I_{1}}^{(0)}\right\},
$$


which determine $\mu_{\alpha}$ and $\bar{\mu}_{\alpha}$ once $\tilde{\nu}_{I_{1}}^{(0)}$ are fixed. Since the consistency condition for $\tilde{\Psi}_{I_{1}}^{(0)}$ is given by

$$
0 \approx \dot{\tilde{\Psi}}_{I_{1}}^{(0)}=\left\{\tilde{\Psi}_{I_{1}}^{(0)}, H\right\}+\tilde{\nu}_{J_{1}}^{(0)}\left\{\tilde{\Psi}_{I_{1}}^{(0)}, \tilde{\Psi}_{J_{1}}^{(0)}\right\}
$$

we impose the second degeneracy condition as

$$
\left\{\tilde{\Psi}_{I_{1}}^{(0)}, \tilde{\Psi}_{J_{1}}^{(0)}\right\}=0
$$

and we obtain secondary constraints

$$
\tilde{\Upsilon}_{I_{1}}^{(1)} \equiv-\left\{\tilde{\Psi}_{I_{1}}^{(0)}, H\right\}=\tilde{P}_{I_{1}}^{(1)}-G_{I_{1}}^{(1)}\left(p^{i}, x\right) \approx 0,
$$

where

$$
\tilde{\Upsilon}_{I_{1}}^{(1)} \equiv\left(\Upsilon_{10}^{i_{1}}, \Upsilon_{21}^{i_{2}}, \cdots, \Upsilon_{d, d-1}^{i_{d}}\right)
$$

Recalling that this notation allows us to isolate the first argument as $\tilde{\Upsilon}_{I_{1}}^{(1)}=\left(\Upsilon_{10}^{i_{1}}, \tilde{\Upsilon}_{I_{2}}^{(1)}\right)$, the consistency condition for $\tilde{\Upsilon}_{I_{1}}^{(1)} \approx 0$ is given by

$$
\begin{aligned}
& 0 \approx \dot{\Upsilon}_{10}^{i_{1}}=\left\{\Upsilon_{10}^{i_{1}}, H\right\}+\tilde{\nu}_{J_{1}}^{(0)}\left\{\Upsilon_{10}^{i_{1}}, \tilde{\Psi}_{J_{1}}^{(0)}\right\} \\
& 0 \approx \dot{\tilde{\Upsilon}}_{I_{2}}^{(1)}=\left\{\tilde{\Upsilon}_{I_{2}}^{(1)}, H\right\}+\tilde{\nu}_{J_{1}}^{(0)}\left\{\tilde{\Upsilon}_{I_{2}}^{(1)}, \tilde{\Psi}_{J_{1}}^{(0)}\right\} .
\end{aligned}
$$

Since $\Upsilon_{10}^{i_{1}}$ fixes $P_{10}^{i_{1}}$ or the lowest problematic momentum for $\phi^{i_{1}}$ sector, we would like to avoid generating further constraints from $\dot{\Upsilon}_{10}^{i_{1}} \approx 0$. In other words, we do not need further constraint as the Hamiltonian does not contain linear term such as $Q_{10}^{i_{1}} P$ with some momentum $P$. Therefore the first equation of (4.22) gives $n_{1}$ equations between $\tilde{\nu}_{J_{1}}^{(0)}$. In contrast, we would like to have further constraints from $\dot{\tilde{\Upsilon}}_{I_{2}}^{(1)} \approx 0$ to eliminate remaining linear terms coming from $\phi^{i_{k}}$ sectors with $k \geq 2$. We thus impose the third degeneracy condition

$$
\left\{\tilde{\Upsilon}_{I_{2}}^{(1)}, \tilde{\Psi}_{J_{1}}^{(0)}\right\}=0
$$

As we have discussed in (3.28), this is not the most general condition for (4.22) to determine only $n_{1}$ component of $\tilde{\nu}_{I_{1}}^{(0)}$. Analysis in more general case is definitely interesting, but becomes highly complicated and is beyond the scope of this paper. Thus, we impose (4.23). Then, the second equation of (4.22) yields the tertiary constraints

$$
\tilde{\Upsilon}_{I_{2}}^{(2)} \equiv-\left\{\tilde{\Upsilon}_{I_{2}}^{(1)}, H\right\}=\tilde{P}_{I_{2}}^{(2)}-\tilde{G}_{I_{2}}^{(2)}\left(p^{i}, x\right) \approx 0 .
$$

By induction, for the constraints

$$
\tilde{\Upsilon}_{I_{k}}^{(k)} \equiv-\left\{\tilde{\Upsilon}_{I_{k}}^{(k-1)}, H\right\}=\tilde{P}_{I_{k}}^{(k)}-\tilde{G}_{I_{k}}^{(k)}\left(p^{i}, x\right) \approx 0,
$$

we decompose the consistency conditions as

$$
\begin{aligned}
& 0 \approx \dot{\Upsilon}_{k 0}^{i_{k}}=\left\{\Upsilon_{k 0}^{i_{k}}, H\right\}+\tilde{\nu}_{J_{1}}^{(0)}\left\{\Upsilon_{k 0}^{i_{k}}, \tilde{\Psi}_{J_{1}}^{(0)}\right\}, \\
& 0 \approx \tilde{\tilde{\Upsilon}}_{I_{k+1}}^{(k)}=\left\{\tilde{\Upsilon}_{I_{k+1}}^{(k)}, H\right\}+\tilde{\nu}_{J_{1}}^{(0)}\left\{\tilde{\Upsilon}_{I_{k+1}}^{(k)}, \tilde{\Psi}_{J_{1}}^{(0)}\right\},
\end{aligned}
$$


and impose the degeneracy conditions

$$
\left\{\tilde{\Upsilon}_{I_{k+1}}^{(k)}, \tilde{\Psi}_{J_{1}}^{(0)}\right\}=0
$$

to obtain the constraints

$$
\tilde{\Upsilon}_{I_{k+1}}^{(k+1)} \equiv-\left\{\tilde{\Upsilon}_{I_{k+1}}^{(k)}, H\right\}=\tilde{P}_{I_{k+1}}^{(k+1)}-\tilde{G}_{I_{k+1}}^{(k+1)}\left(p^{i}, x\right) \approx 0,
$$

for $k=2, \cdots, d-1$.

The constraints (4.20), (4.24), (4.25), (4.28) form a matrix

$$
\Upsilon \equiv \boldsymbol{P}-\boldsymbol{G} \approx 0
$$

where

$$
\Upsilon \equiv\left(\begin{array}{cccc}
\Upsilon_{10}^{i_{1}} & & & \\
\Upsilon_{20}^{i_{2}} & \Upsilon_{21}^{i_{2}} & & \\
\vdots & & \ddots & \\
\Upsilon_{d 0}^{i_{d}} & \Upsilon_{d 1}^{i_{d}} & \cdots & \Upsilon_{d, d-1}^{i_{d}}
\end{array}\right), \quad \boldsymbol{G} \equiv\left(\begin{array}{cccc}
G_{10}^{i_{1}} & & & \\
G_{20}^{i_{2}} & G_{21}^{i_{2}} & & \\
\vdots & & \ddots & \\
G_{d 0}^{i_{d}} & G_{d 1}^{i_{d}} & \cdots & G_{d, d-1}^{i_{d}}
\end{array}\right)
$$

We then arrive at the consistency condition for the last constraint $\tilde{\Upsilon}_{I_{d}}^{(d)}=\Upsilon_{d 0}^{i_{d}}$

$$
0 \approx \dot{\Upsilon}_{d 0}^{i_{d}}=\left\{\Upsilon_{d 0}^{i_{d}}, H\right\}+\tilde{\nu}_{J_{1}}^{(0)}\left\{\Upsilon_{d 0}^{i_{d}}, \tilde{\Psi}_{J_{1}}^{(0)}\right\}
$$

After the above procedure, the constraints (4.29) fix the linear momentum terms in the Hamiltonian (4.16), and we are left with the consistency conditions

$$
\left\{\Upsilon_{k 0}^{i_{k}}, H\right\}+\tilde{\nu}_{I_{1}}^{(0)}\left\{\Upsilon_{k 0}^{i_{k}}, \tilde{\Psi}_{I_{1}}^{(0)}\right\} \approx 0, \quad(k=1, \cdots, d) .
$$

Therefore, if this set of equations determine the Lagrange multipliers $\tilde{\nu}_{I_{1}}^{(0)}$, we complete the Dirac algorithm.

In parallel to (3.32), we can list all the phase space variables of the current system (4.4) as

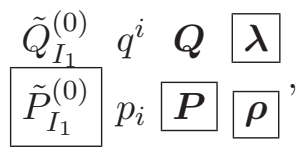

where the boxed variables are fixed in terms of other variables via constraints obtained so far. Nevertheless, as a natural generalization of the results obtained in section 3, we are interested in the case where the number of degrees of freedom matches the number of variables by removing all the ghosts associated with the canonical variables which correspond to the higher-than-first time derivatives of the original variables, and all the constraints are second class. Such canonical variables come from $\tilde{Q}_{I_{1}}^{(0)} \equiv\left(Q_{11}^{i_{1}}, Q_{22}^{i_{2}}, \cdots, Q_{d d}^{i_{d}}\right)$ and $\boldsymbol{Q}$. We can combine $\tilde{Q}_{I_{1}}^{(0)}$ and $\boldsymbol{Q}$, and list up them as a larger matrix

$$
\left(\begin{array}{cccccc}
Q_{10}^{i_{1}} & Q_{11}^{i_{1}} & & & & \\
Q_{20}^{i_{2}} & Q_{21}^{i_{2}} & Q_{22}^{i_{2}} & & & \\
Q_{30}^{i_{3}} & Q_{31}^{i_{3}} & Q_{32}^{i_{3}} & Q_{33}^{i_{3}} & & \\
\vdots & & & & \ddots & \\
Q_{d 0}^{i_{d}} & Q_{d 1}^{i_{d}} & Q_{d 2}^{i_{d}} & \cdots & Q_{d, d-1}^{i_{d}} & Q_{d d}^{i_{d}}
\end{array}\right)
$$


The first two columns are the original variables and their first-order time derivatives, and the remaining part

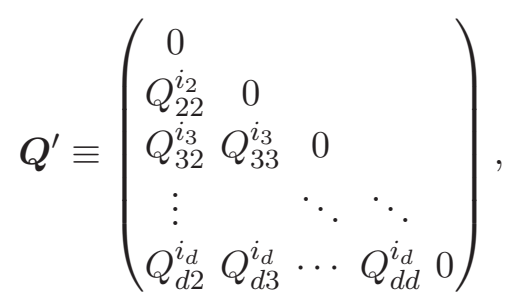

is the variables that we would like to fix by invoking additional constraints. Here, we keep a row and a column of zeros in the definition of $\boldsymbol{Q}^{\prime}$ and make its dimension as the same as the other matrices denoted by the bold font.

We thus require an additional degeneracy condition

$$
\left\{\Upsilon_{k 0}^{i_{k}}, \tilde{\Psi}_{I_{1}}^{(0)}\right\}=0, \quad(k=2, \cdots, d),
$$

with which (4.32) yields additional constraints

$$
\Omega_{k 0}^{i_{k}} \equiv-\left\{\Upsilon_{k 0}^{i_{k}}, H\right\} \approx 0, \quad(k=2, \cdots, d) .
$$

Note that, analogous to section 3 , we do not impose the degeneracy condition for $i_{1}$ component. The number of the constraints is $n_{2}+\cdots n_{d}$, which is the same as the number of the nonvanishing components of the first column of $\boldsymbol{Q}^{\prime}$ in (4.35). The consistency conditions for $\Omega_{k 0}^{i_{k}}$ are given by

$$
\left\{\Omega_{k 0}^{i_{k}}, H\right\}+\tilde{\nu}_{I_{1}}^{(0)}\left\{\Omega_{k 0}^{i_{k}}, \tilde{\Psi}_{I_{1}}^{(0)}\right\} \approx 0, \quad(k=2, \cdots, d) .
$$

To obtain a sufficient number of constraints, we further impose degeneracy conditions for $k=3, \cdots, d$

$$
\left\{\Omega_{k 0}^{i_{k}}, \tilde{\Psi}_{I_{1}}^{(0)}\right\}=0, \quad(k=3, \cdots, d),
$$

and we obtain constraints

$$
\Omega_{k 1}^{i_{k}} \equiv-\left\{\Omega_{k 0}^{i_{k}}, H\right\} \approx 0, \quad(k=3, \cdots, d),
$$

whose consistency conditions are given by

$$
\left\{\Omega_{k 1}^{i_{k}}, H\right\}+\tilde{\nu}_{I_{1}}^{(0)}\left\{\Omega_{k 1}^{i_{k}}, \tilde{\Psi}_{I_{1}}^{(0)}\right\} \approx 0, \quad(k=3, \cdots, d) .
$$

We continue the process $k-2$ times and impose

$$
\left\{\Omega_{\ell k}^{i_{\ell}}, \tilde{\Psi}_{I_{1}}^{(0)}\right\}=0, \quad(k=3, \cdots, d-3 ; \ell=k+1, \cdots, d),
$$

until we obtain a set of constraints

$$
\boldsymbol{\Omega} \equiv\left(\begin{array}{ccccc}
0 & & & & \\
\Omega_{20}^{i_{2}} & 0 & & & \\
\Omega_{30}^{i_{3}} & \Omega_{31}^{i_{3}} & 0 & & \\
\vdots & & \ddots & \ddots & \\
\Omega_{d 0}^{i_{d}} & \Omega_{d 1}^{i_{d}} & \cdots & \Omega_{d, d-2}^{i_{d}} & 0
\end{array}\right)
$$


which precisely corresponds to $\boldsymbol{Q}^{\prime}$ in (4.35). The remaining consistency conditions corresponding to the diagonal zero components of (4.43) are given by

$$
\left\{\hat{\Omega}_{I_{1}}^{(1)}, H\right\}+\tilde{\nu}_{I_{1}}^{(0)}\left\{\hat{\Omega}_{I_{1}}^{(1)}, \tilde{\Psi}_{I_{1}}^{(0)}\right\} \approx 0,
$$

where we defined

$$
\hat{\Omega}_{I_{1}}^{(1)} \equiv\left(\Upsilon_{10}^{i_{1}}, \tilde{\Omega}_{I_{2}}^{(2)}\right)
$$

and

$$
\tilde{\Omega}_{I_{2}}^{(2)} \equiv\left(\Omega_{20}^{i_{2}}, \Omega_{31}^{i_{3}}, \cdots, \Omega_{d, d-2}^{i_{d}}\right) .
$$

All the degeneracy conditions we imposed above are (4.9), (4.19), (4.23), (4.27), (4.36), (4.39), (4.42), which are summarized as

$$
\begin{aligned}
& L_{I_{1} J_{1}}-L_{I_{1} i} L^{i j} L_{j J_{1}}=0, \\
& \left\{\tilde{\Psi}_{I_{1}}^{(0)}, \tilde{\Psi}_{J_{1}}^{(0)}\right\}=0, \\
& \left\{\boldsymbol{\Upsilon}, \tilde{\Psi}_{J_{1}}^{(0)}\right\}=0 \quad \text { except } \quad \Upsilon_{10}^{i_{1}}, \\
& \left\{\boldsymbol{\Omega}, \tilde{\Psi}_{J_{1}}^{(0)}\right\}=0 \quad \text { except } \quad \tilde{\Omega}_{I_{2}}^{(2)} \text {. }
\end{aligned}
$$

Now we require (4.44) determines all the Lagrange multipliers $\tilde{\nu}_{I_{1}}^{(0)}$, and complete the Dirac algorithm. As a generalization of (3.39), we define a matrix

$$
Z_{I_{1} J_{1}} \equiv\left\{\hat{\Omega}_{I_{1}}^{(1)}, \tilde{\Psi}_{J_{1}}^{(0)}\right\}
$$

One can show that $Z_{i_{k}, j_{\ell}}=0$ for $k<\ell$ [see also (4.56) below]. Thus, the necessary and sufficient condition for (4.44) to determine all $\tilde{\nu}_{I_{1}}^{(0)}$ is that each $\left(i_{k}, j_{k}\right)$ submatrix is nondegenerate

$$
\operatorname{det} Z_{i_{k} j_{k}} \neq 0
$$

With the degeneracy conditions (4.47), we obtain the constraints $\left(\mathbf{\Phi}, \overline{\mathbf{\Phi}}, \tilde{\Psi}_{I_{1}}^{(0)}, \mathbf{\Upsilon}, \boldsymbol{\Omega}\right)$ which are given in (4.8), (4.10), (4.29), (4.43). The correspondence between the canonical variables and the constraints that fix them are

$$
\lambda: \boldsymbol{\Phi}, \quad \boldsymbol{\rho}: \overline{\boldsymbol{\Phi}}, \quad \tilde{P}_{I_{1}}^{(0)}: \tilde{\Psi}_{I_{1}}^{(0)}, \quad \boldsymbol{P}: \Upsilon, \quad \boldsymbol{Q}^{\prime}: \boldsymbol{\Omega} .
$$

While we do not show that the constraints $\Omega$ fixes the variables $\boldsymbol{Q}^{\prime}$ explicitly, the correspondence is reasonable as the $\boldsymbol{Q}^{\prime}$ amounts to higher derivatives and the number of constraints and variables precisely match. The number of constraints are respectively

$$
\boldsymbol{\Phi}: \sum_{k=1}^{d} k n_{k}, \quad \overline{\mathbf{\Phi}}: \sum_{k=1}^{d} k n_{k}, \quad \tilde{\Psi}_{I_{1}}^{(0)}: \sum_{k=1}^{d} n_{k}, \quad \boldsymbol{\Upsilon}: \sum_{k=1}^{d} k n_{k}, \quad \boldsymbol{\Omega}: \sum_{k=1}^{d}(k-1) n_{k} .
$$

To count the number of degrees of freedom we shall classify them into first class and second class constraints.

While the correspondence to canonical variables is transparent for the combination $\left(\boldsymbol{\Phi}, \overline{\boldsymbol{\Phi}}, \tilde{\Psi}_{I_{1}}^{(0)}, \Upsilon, \boldsymbol{\Omega}\right)$, it is not the best combination for counting the number of degrees of 
freedom as the Dirac matrix is not simple. Let us focus on $\left(\tilde{\Psi}_{I_{1}}^{(0)}, \boldsymbol{\Upsilon}, \boldsymbol{\Omega}\right)$ and consider a more useful basis. These constraints are connected each other by chains of Poisson brackets. Let us list them as

$$
\begin{aligned}
& \Psi_{11}^{i_{1}} \mid \Upsilon_{10}^{i_{1}} \\
& \Psi_{22}^{i_{2}} \Upsilon_{21}^{i_{2}} \Upsilon_{20}^{i_{2}} \Omega_{20}^{i_{2}} \\
& \Psi_{33}^{i_{3}} \Upsilon_{32}^{i_{3}} \Upsilon_{31}^{i_{3}} \Upsilon_{30}^{i_{3}} \Omega_{30}^{i_{3}} \Omega_{31}^{i_{3}} \\
& \Psi_{44}^{i_{4}} \Upsilon_{43}^{i_{4}} \Upsilon_{42}^{i_{4}} \Upsilon_{41}^{i_{4}} \Upsilon_{40}^{i_{4}} \Omega_{40}^{i_{4}} \Omega_{41}^{i_{4}} \Omega_{42}^{i_{4}}
\end{aligned}
$$

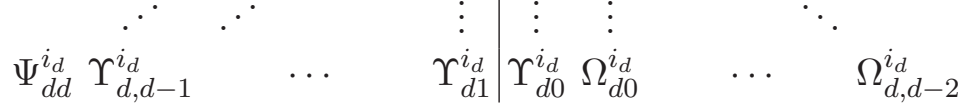

Each row is connected by a chain of Poisson brackets. Starting from the most left component of $\tilde{\Psi}_{I_{1}}^{(0)}$, the next right component is defined by taking a Poisson bracket with $-H$, and we continue to proceed to the right component until we arrive at the most right component of $\hat{\Omega}_{I_{1}}^{(1)}$ ending with nonvanishing Poisson bracket with corresponding component of $\tilde{\Psi}_{I_{1}}^{(0)}$. Taking the bottom row of (4.52) as an example, we have $\Upsilon_{d, d-1}^{i_{d}}=-\left\{\Psi_{d d}^{i_{d}}, H\right\}$, $\Upsilon_{d, d-2}^{i_{d}}=-\left\{\Upsilon_{d, d-1}^{i_{d}}, H\right\}, \cdots, \Omega_{d, d-2}^{i_{d}}=-\left\{\Omega_{d, d-3}^{i_{d}}, H\right\}$, and $\operatorname{det}\left\{\Omega_{d, d-2}^{i_{d}}, \Psi_{d d}^{i_{d}}\right\} \neq 0$. To make use of the structure of Poisson brackets, it is more useful to divide the constraints by the vertical line shown in (4.52) rather than distinguishing them by $\Upsilon, \Omega$ notation. We thus reclassify and relabel them as

$$
\begin{aligned}
& \Psi_{11}^{i_{1}} \mid \Upsilon_{10}^{i_{1}} \\
& \begin{array}{ll|ll}
\Psi_{22}^{i_{2}} & A_{1}^{i_{2}} & B_{1}^{i_{2}} & \Omega_{20}^{i_{2}}
\end{array} \\
& \begin{array}{lll|lll}
\Psi_{33}^{i_{3}} & A_{1}^{i_{3}} & A_{2}^{i_{3}} & B_{2}^{i_{3}} & B_{1}^{i_{3}} & \Omega_{31}^{i_{3}}
\end{array} \\
& \begin{array}{lllllllll}
\Psi_{44}^{i_{4}} & A_{1}^{i_{4}} & A_{2}^{i_{4}} & A_{3}^{i_{4}} & B_{3}^{i_{4}} & B_{2}^{i_{4}} & B_{1}^{i_{4}} & \Omega_{42}^{i_{4}}
\end{array}
\end{aligned}
$$

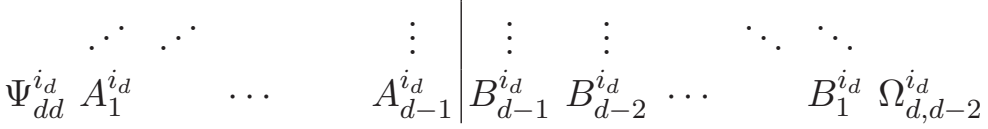

The chains of Poisson brackets are then rewritten as

$$
\begin{aligned}
\Upsilon_{10}^{i_{1}} & =-\left\{\Psi_{11}^{i_{1}}, H\right\}, & & \\
A_{1}^{i_{k}} & =-\left\{\Psi_{k k}^{i_{k}}, H\right\}, & & (k=2, \cdots, d), \\
A_{a+1}^{i_{k}} & =-\left\{A_{a}^{i_{k}}, H\right\}, & & (k=3, \cdots, d ; a=1, \cdots, k-2), \\
B_{k-1}^{i_{k}} & =-\left\{A_{k-1}^{i_{k}}, H\right\}, & & (k=2, \cdots, d), \\
B_{a}^{i_{k}} & =-\left\{B_{a+1}^{i_{k}}, H\right\}, & & (k=3, \cdots, d ; a=1, \cdots, k-2), \\
\Omega_{k, k-2}^{i_{k}} & =-\left\{B_{1}^{i_{k}}, H\right\}, & & (k=2, \cdots, d) .
\end{aligned}
$$

Note that the last two degeneracy conditions of (4.47) read

$$
\left\{A_{a}^{i_{k}}, \tilde{\Psi}_{I_{1}}^{(0)}\right\}=\left\{B_{a}^{i_{k}}, \tilde{\Psi}_{I_{1}}^{(0)}\right\}=0, \quad(a=1, \cdots, k-1) .
$$

From these relations and the Jacobi identity, one can show that

$$
\begin{aligned}
& \left\{A_{a}^{i_{k}}, B_{a}^{j_{k}}\right\}=(-1)^{a+1} Z_{j_{k} i_{k}}, \\
& \left\{A_{a}^{i_{k}}, B_{b}^{j_{\ell}}\right\}=0, \quad(a<b \text { or } 2 \ell-b<2 k-a), \\
& \left\{A_{a}^{i_{k}}, A_{b}^{j_{\ell}}\right\}=0 .
\end{aligned}
$$


With the above basis, the Dirac matrix is given by

\begin{tabular}{l|ccccccccc} 
& $\boldsymbol{\Phi}$ & $\overline{\mathbf{\Phi}}$ & $\tilde{\Psi}$ & $\hat{\Omega}$ & $A_{1}^{j_{2}}$ & $B_{1}^{j_{2}}$ & $\cdots$ & $A_{d-1}^{j_{d}}$ & $B_{d-1}^{j_{d}}$ \\
\hline $\mathbf{\Phi}$ & 0 & $-\mathbf{1}$ & $*$ & $*$ & $*$ & $*$ & $*$ & $*$ & $*$ \\
$\overline{\mathbf{\Phi}}$ & $\mathbf{1}$ & 0 & 0 & 0 & 0 & 0 & 0 & 0 & 0 \\
$\tilde{\Psi}$ & $*$ & 0 & 0 & $-Z^{T}$ & 0 & 0 & 0 & 0 & 0 \\
$\hat{\Omega}$ & $*$ & 0 & $Z$ & $*$ & $*$ & $*$ & $*$ & $*$ & $*$ \\
$A_{1}^{i_{2}}$ & $*$ & 0 & 0 & $*$ & 0 & $Z_{j_{2} i_{2}}$ & 0 & 0 & 0 \\
$B_{1}^{i_{2}}$ & $*$ & 0 & 0 & $*$ & $-Z_{i_{2} j_{2}}$ & $*$ & $*$ & $*$ & $*$ \\
$\vdots$ & $*$ & 0 & 0 & $*$ & 0 & $*$ & $\ddots$ & $\vdots$ & $\vdots$ \\
$A_{d-1}^{i_{d}}$ & $*$ & 0 & 0 & $*$ & 0 & $*$ & $\cdots$ & 0 & $(-1)^{d} Z_{j_{d} i_{d}}$ \\
$B_{d-1}^{i_{d}}$ & $*$ & 0 & 0 & $*$ & 0 & $*$ & $\cdots$ & $(-1)^{d+1} Z_{i_{d} j_{d}}$ & $*$
\end{tabular}

The determinant of the Dirac matrix is thus given by

$$
\left(\operatorname{det} Z_{i_{1} j_{1}}\right)^{2} \prod_{k=2}^{d}\left(\operatorname{det} Z_{i_{k} j_{k}}\right)^{4},
$$

which is nonvanishing by virtue of (4.49). Hence, all the constraints are second class, whose total number is given by summing up (4.51)

$$
N_{2 \text { nd }}=4 \sum_{k=0}^{d} k n_{k} .
$$

Using (4.6), the number of degrees of freedom is

$$
N_{\text {DOF }}=\frac{1}{2}\left(N_{\text {can }}-N_{2 \text { nd }}\right)=\sum_{k=0}^{d} n_{k} .
$$

\subsection{Euler-Lagrange equation}

The Euler-Lagrange equation for the Lagrangian (4.15) can be written as

$$
\begin{array}{rlrl}
\dot{L}_{i}-L_{q^{i}} & =0, & & \\
\dot{L}_{I_{1}}-L_{\tilde{Q}_{I_{1}}^{(0)}}^{(0)} \tilde{\lambda}_{I_{1}}^{(1)} & =0, & & \\
L_{\tilde{Q}_{I_{k+1}}^{(k)}-\tilde{\lambda}_{I_{k+1}}^{(k+1)}-\dot{\tilde{\lambda}}_{I_{k+1}}^{(k)}}=0, & & (k=1, \cdots, d-1), \\
L_{Q_{k 0}^{i_{k}}-\dot{\lambda}_{k 0}^{i_{k}}}=0, & & (k=1, \cdots, d), \\
\tilde{Q}_{I_{k+1}}^{(k)}-\dot{\tilde{Q}}_{I_{k+1}}^{(k+1)} & =0, & & (k=0, \cdots, d-1), \\
Q_{k 1}^{i_{k}}-\dot{Q}_{k 0}^{i_{k}} & =0, & & (k=1, \cdots, d),
\end{array}
$$

where we recall that $L_{i} \equiv L_{\dot{q}^{i}}$ and $L_{I_{1}} \equiv L_{\tilde{Q}_{I_{1}}^{(0)}}$. The above equations (4.61)-(4.66) have the same structure as the equations (3.46)-(3.53), though the numbers of two sets of equations 
are different: (4.61), (4.62), (4.63), (4.65) correspond to (3.46), (3.47), (3.48), (3.51), respectively, whereas (4.64) corresponds to (3.49) and (3.50), and (4.66) corresponds to (3.52) and (3.53). We shall show below that their reduction to a second-order system is a natural generalization of the analysis in section 3.2.

Following section 3.2, first we focus on $\tilde{\lambda}_{I_{1}}^{(1)}$ in (4.62). While a priori (4.62) implies that $\tilde{\lambda}_{I_{1}}^{(1)}$ depends on $\ddot{Q}_{I_{1}}^{(0)}$, with the first degeneracy condition (4.9) or the additional primary constraints (4.10), we can show that (4.61) and (4.62) read

$$
\begin{aligned}
\tilde{\lambda}_{I_{1}}^{(1)} & =L_{\tilde{Q}_{I_{1}}^{(0)}}-\tilde{F}_{I_{1} p_{i}}^{(0)} L_{q^{i}}-\dot{x} \tilde{F}_{I_{1} x}^{(0)}, \\
\mathcal{E}_{i} & \equiv \ddot{q}^{i}+F_{I_{1} p_{i}} \ddot{\tilde{Q}}_{I_{1}}^{(0)}-L^{i j}\left(L_{q^{j}}-\dot{x} L_{j x}\right)=0,
\end{aligned}
$$

the former of which corresponds to the secondary constraints (4.20).

Next we focus on (4.63) and (4.64) with $k=1$. We take a time derivative of (4.67), $I_{2}$ component of which gives $\tilde{\lambda}_{I_{2}}^{(2)}$ from (4.63) with $k=1$, and $i_{1}$ component of which gives EOM for $Q_{10}^{i_{1}}$ from (4.64) with $k=1$. Again, while they a priori depend on $\ddot{\tilde{Q}}_{I_{1}}^{(0)}$, with the second degeneracy condition (4.19), (4.67) implies $\tilde{\lambda}_{I_{1}}^{(1)}=\tilde{G}_{I_{1}}^{(1)}\left(L_{i}, x\right)$ and $\ddot{\tilde{Q}}_{I_{1}}^{(0)}$ dependences identically vanish. From (4.63) with $k=1$ we obtain

$$
\tilde{\lambda}_{I_{2}}^{(2)}=L_{\tilde{Q}_{I_{2}}^{(1)}}-\tilde{G}_{I_{2} p_{i}}^{(1)} L_{q^{i}}-\dot{x} \tilde{G}_{I_{2} x}^{(1)},
$$

which corresponds to the tertiary constraints $\tilde{\lambda}_{I_{2}}^{(2)}=G_{I_{2}}^{(2)}\left(L_{i}, x\right)$ in (4.24). Also, from (4.64) with $k=1$ we obtain EOM for $Q_{10}^{i_{1}}$ as

$$
0=\mathcal{E}_{10}^{i_{1}} \equiv L_{Q_{10}^{i_{1}}}-G_{10, p_{i}}^{i_{1}} L_{q^{i}}-\dot{x} G_{10, x}^{i_{1}}=-\left\{\Upsilon_{10}^{i_{1}}, H\right\}
$$

which, recalling the notation (4.3), is the EOM for $\phi^{i_{1}}$, and shows that the first term of the most right hand side of the first equation of (4.22) vanishes.

Inductively, for $k=2, \cdots d-1$, by using a time derivative of $\tilde{\lambda}_{I_{k}}^{(k)}=\tilde{G}_{I_{k}}^{(k)}\left(L_{i}, x\right)$ and the degeneracy conditions (4.23) and (4.27), we can reduce (4.63) and (4.64) and obtain

$$
\begin{aligned}
& \tilde{\lambda}_{I_{k+1}}^{(k+1)}=L_{\tilde{Q}_{I_{k+1}}^{(k)}}-\tilde{G}_{I_{k+1} p_{i}}^{(k)} L_{q^{i}}-\dot{x} \tilde{G}_{I_{k+1} x}^{(k)} \equiv \tilde{G}_{I_{k+1}}^{(k+1)}\left(L_{i}, x\right), \\
& 0=\mathcal{E}_{k 0}^{i_{k}} \equiv L_{Q_{k 0}^{i_{k}}}-G_{k 0, p_{i}}^{i_{k}} L_{q^{i}}-\dot{x} G_{k 0, x}^{i_{k}}=-\left\{\Upsilon_{k 0}^{i_{k}}, H\right\},
\end{aligned}
$$

the latter of which is the EOM for $\phi^{i_{k}}$ and related to (4.26). Finally, plugging a time derivative of (4.71) into (4.64) with $k=d$ and using the degeneracy condition (4.27) with $k=d-1$, we obtain EOM for $Q_{d 0}^{i_{d}}=\phi^{i_{d}}$ as

$$
0=\mathcal{E}_{d 0}^{i_{d}} \equiv L_{Q_{d 0}^{i_{d}}}-G_{d 0, p_{i}}^{i_{d}} L_{q^{i}}-\dot{x} G_{d 0, x}^{i_{d}}=-\left\{\Upsilon_{d 0}^{i_{d}}, H\right\}
$$

which is related to (4.31).

We thus obtain EOMs for $q^{i}, \phi^{i_{1}}, \cdots, \phi^{i_{d}}$ as (4.68), (4.72), (4.73), but they still contain higher derivatives. We can construct a set of EOMs with derivatives up to second-order as follows. By virtue of the degeneracy condition $(4.36), \mathcal{E}_{k 0}^{i_{k}}$ for $k=2, \cdots, d$ are functions of 
$\left(L_{i}, x\right)$ and thus a time derivative of EOMs $\mathcal{E}_{k 1}^{i_{k}} \equiv \dot{\mathcal{E}}_{k 0}^{i_{k}}$ for $k=2, \cdots, d$ does not contain $\ddot{\widetilde{Q}}_{I_{1}}^{(0)}$. We continue this procedure with the degeneracy conditions (4.39) and (4.42) to obtain a set of EOMs

$$
0=\mathcal{E} \equiv\left(\begin{array}{cccc}
\mathcal{E}_{10}^{i_{1}} & & & \\
\mathcal{E}_{20}^{i_{2}} & \mathcal{E}_{21}^{i_{2}} & & \\
\vdots & & \ddots & \\
\mathcal{E}_{d 0}^{i_{d}} & \mathcal{E}_{d 1}^{i_{d}} & \cdots & \mathcal{E}_{d, d-1}^{i_{d}}
\end{array}\right)
$$

Generalizing the logic for (3.60)-(3.62), we expect that in general the condition (4.49) guarantees that we can solve (4.74) and express

$$
\begin{aligned}
\dot{\tilde{Q}}_{I_{1}}^{(0)} & =\tilde{F}_{I_{1}}^{(0)}\left(\dot{q}^{i}, q^{i}, Q_{k 0}^{i_{k}}, Q_{k 1}^{i_{k}}\right), \\
\boldsymbol{Q}^{\prime} & =\boldsymbol{F}^{\prime}\left(\dot{q}^{i}, q^{i}, Q_{k 0}^{i_{k}}, Q_{k 1}^{i_{k}}\right),
\end{aligned}
$$

where $\boldsymbol{F}^{\prime}$ is a matrix with nonvanishing arguments corresponding to $\boldsymbol{Q}^{\prime}$ defined in (4.35). These equations are a generalization of (3.60)-(3.62). From these equations, $\dot{Q}_{11}^{i_{1}}=\ddot{\phi}^{i_{1}}$ and $Q_{k 2}^{i_{k}}=\ddot{\phi}^{i_{k}}$ for $k=2, \cdots, d$ can be written down in terms of derivatives up to first order. Taking a time derivative of (4.75) and plugging (4.75) and (4.76) we obtain

$$
\ddot{\tilde{Q}}_{I_{1}}^{(0)}=\tilde{F}_{I_{1}}^{(0)}\left(\ddot{q}^{i}, \dot{q}^{i}, q^{i}, Q_{k 0}^{i_{k}}, Q_{k 1}^{i_{k}}\right)
$$

By substituting (4.75)-(4.77) to (4.68) we obtain EOM containing at most $\ddot{q}^{i}$. We thus obtain a system of $\sum_{k=0}^{d} n_{k}$ EOMs that contain at most second-order derivatives.

\section{Conclusions and discussion}

In this paper, we have clarified how to construct no-ghost theory for general Lagrangians for point particle system involving arbitrary higher-order time derivatives. The first no-ghost theory involving third-order derivative was the quadratic model studied in [21]. In section 2, we provided the specific no-ghost theory that involves arbitrary higher-order derivative. Then, in section 3, we have derived the conditions for general Lagrangian involving thirdorder derivatives to possess only healthy DOFs. As shown in [21], in sharp contrast to theories with up to the second-order time derivatives in the Lagrangian, eliminating linear dependence of canonical momenta in the Hamiltonian is not sufficient for those with higherthan-second-order derivatives, and that canonical coordinates corresponding to the higher time-derivatives also need to be removed appropriately. In [21], this process was confirmed for the quadratic model, and in section 3 we confirmed it for any Lagrangian involving thirdorder derivatives. We have also shown that, as long as these conditions are satisfied, the Euler-Lagrange equations can be reduced to a system of second-order differential equations, which is consistent with the absence of ghost DOFs. Finally, in section 4 we have extended these analyses to general theories involving arbitrary higher-order derivatives. The caveat is that we have concentrated on the cases, in which all of the constraints are second class. If some of them are first class, the analyses would be much more complicated and case-bycase analysis would be necessary though such analyses are indispensable for gauge theories. 
Nevertheless, by introducing adequate gauge fixing terms, first class constraints turn into second class ones, to which the analysis in the present paper would apply. We leave this kind of analysis as future work.

While our analysis is confined to the analytic mechanics for a system of point particles as the first step, it clarifies the essence of the construction of degenerate theories, and it is quite robust as they apply to any Lagrangian involving arbitrary higher-order derivatives. Furthermore, the analysis for field theory can be reduced into the one for the analytic mechanics by exploiting ADM decomposition with a choice of direction of time. After that, the result of the present paper will guide us how to construct ghost-free field theories with arbitrary higher-order derivatives. Actually, the extension of our analysis to field theories with arbitrary higher-order derivatives is quite interesting, for example, scalar (and vector) fields in the Minkowski background, scalar-tensor theories, vector-tensor theories, scalar-vector-tensor (TeVeS) theories, and even a theory with fermionic degrees of freedom. Especially, it is challenging to find a healthy theory with higher-order derivative terms, which cannot be transformed to a theory with only up to first order derivatives by invertible transformation [29]. We also leave all of these topics as future work.

\section{Acknowledgments}

This work was supported in part by JSPS KEKENHI Grant Numbers, JP17H06359 (H.M.), JP18K13565 (H.M.), JP15K17632 (T.S.), JP17H06359 (T.S.), JP15H05888 (T.S. \& M.Y.), JP25287054 (M.Y.), JP18H04579 (M.Y.).

\section{A Lagrangian with single third-order derivative}

In this appendix, we consider the special case of the Lagrangian considered in section 3 with $\mathcal{N}=1, \mathcal{A}=0$. In this case some part of degeneracy conditions are identically satisfied. While it is obvious that the equation corresponding to the second degeneracy conditions (3.20) is identically satisfied as $\{\Psi, \Psi\}=0$, it is more subtle to see the another equation $\{\Lambda, \Psi\}=0$ corresponding to the fourth degeneracy condition (3.34) is identically satisfied. Below we provide the proof of this equation.

The consistency condition for the tertiary constraint corresponding to (3.33) reads

$$
0 \approx \dot{\Lambda}=\{\Lambda, H\}+\nu\{\Lambda, \Psi\}
$$

To obtain the quaternary constraint, we need

$$
\{\Lambda, \Psi\}=F_{\psi}-I_{R}+I_{q^{i}} F_{p_{i}}-I_{p_{i}} F_{q^{i}}=0
$$

corresponding to the fourth degeneracy condition (3.34). Actually, we can show that (A.2) 
identically holds by using Jacobi identity repeatedly:

$$
\begin{aligned}
\{\Lambda, \Psi\}= & \left\{-I, P_{Q}-F\right\}-\{\pi, F\} \\
= & \left\{\left\{G, H_{0}+\pi R+P_{R} Q\right\}, P_{Q}-F\right\}-\left\{\left\{P_{R}, H_{0}\right\}, P_{Q}-F\right\}-\{\pi, F\} \\
= & -\left\{\left\{H_{0}+\pi R+P_{R} Q, P_{Q}-F\right\}, G\right\}-\left\{\left\{P_{Q}-F, G\right\}, H_{0}+\pi R+P_{R} Q\right\} \\
& -\left\{\left\{P_{R}, H_{0}\right\}, P_{Q}-F\right\}-\{\pi, F\} \\
= & -\left\{-G+P_{R}, G\right\}-\left\{\left\{P_{R}, F\right\}, H_{0}+\pi R+P_{R} Q\right\}-\left\{\left\{P_{R}, H_{0}\right\}, P_{Q}-F\right\}-\{\pi, F\} \\
= & \left\{G, P_{R}\right\}-\left\{\left\{P_{R}, F\right\}, H_{0}+\pi R+P_{R} Q\right\}-\left\{\left\{P_{R}, H_{0}\right\}, P_{Q}-F\right\}-\{\pi, F\} \\
= & \left\{\left\{P_{Q}, H_{0}\right\}, P_{R}\right\}-\left\{\left\{F, H_{0}\right\}, P_{R}\right\}-\left\{\{F, \pi\} R, P_{R}\right\}-\left\{\left\{F, P_{R}\right\} Q, P_{R}\right\} \\
& -\left\{\left\{P_{R}, F\right\}, H_{0}\right\}-\left\{\left\{P_{R}, F\right\}, \pi R\right\}-\left\{\left\{P_{R}, F\right\}, P_{R} Q\right\} \\
& -\left\{\left\{P_{R}, H_{0}\right\}, P_{Q}\right\}+\left\{\left\{P_{R}, H_{0}\right\}, F\right\}-\{\pi, F\} \\
= & -\{F, \pi\}-\left\{\{F, \pi\}, P_{R}\right\} R-\left\{\left\{P_{R}, F\right\}, \pi\right\} R-\{\pi, F\} \\
= & 0 .
\end{aligned}
$$

We thus have the quaternary constraint

$$
0 \approx \Omega \equiv-\{\Lambda, H\}=-L_{\psi}+I_{p_{i}} L_{q^{i}}+\dot{x} I_{x}
$$

corresponding to (3.35).

Open Access. This article is distributed under the terms of the Creative Commons Attribution License (CC-BY 4.0), which permits any use, distribution and reproduction in any medium, provided the original author(s) and source are credited.

\section{References}

[1] WMAP collaboration, C.L. Bennett et al., Nine-Year Wilkinson Microwave Anisotropy Probe (WMAP) Observations: Final Maps and Results, Astrophys. J. Suppl. 208 (2013) 20 [arXiv: 1212.5225] [INSPIRE].

[2] WMAP collaboration, G. Hinshaw et al., Nine-Year Wilkinson Microwave Anisotropy Probe (WMAP) Observations: Cosmological Parameter Results, Astrophys. J. Suppl. 208 (2013) 19 [arXiv: 1212.5226] [INSPIRE].

[3] Planck collaboration, R. Adam et al., Planck 2015 results. I. Overview of products and scientific results, Astron. Astrophys. 594 (2016) A1 [arXiv:1502.01582] [INSPIRE].

[4] Planck collaboration, P.A.R. Ade et al., Planck 2015 results. XX. Constraints on inflation, Astron. Astrophys. 594 (2016) A20 [arXiv:1502.02114] [INSPIRE].

[5] Supernova Search Team collaboration, A.G. Riess et al., Observational evidence from supernovae for an accelerating universe and a cosmological constant, Astron. J. 116 (1998) 1009 [astro-ph/9805201] [INSPIRE].

[6] Supernova Cosmology Project collaboration, S. Perlmutter et al., Measurements of Omega and Lambda from 42 high redshift supernovae, Astrophys. J. 517 (1999) 565 [astro-ph/9812133] [INSPIRE]. 
[7] M. Ostrogradsky, Mémoires sur les équations différentielles, relatives au problème des isopérimètres, Mem. Acad. St. Petersbourg 6 (1850) 385 [InSPIRE].

[8] G.W. Horndeski, Second-order scalar-tensor field equations in a four-dimensional space, Int. J. Theor. Phys. 10 (1974) 363 [INSPIRE].

[9] C. Deffayet, X. Gao, D.A. Steer and G. Zahariade, From k-essence to generalised Galileons, Phys. Rev. D 84 (2011) 064039 [arXiv:1103.3260] [inSPIRE].

[10] T. Kobayashi, M. Yamaguchi and J. Yokoyama, Generalized G-inflation: Inflation with the most general second-order field equations, Prog. Theor. Phys. 126 (2011) 511 [arXiv: 1105.5723] [INSPIRE].

[11] M. Zumalacárregui and J. García-Bellido, Transforming gravity: from derivative couplings to matter to second-order scalar-tensor theories beyond the Horndeski Lagrangian, Phys. Rev. D 89 (2014) 064046 [arXiv: 1308.4685] [InSPIRE].

[12] J. Gleyzes, D. Langlois, F. Piazza and F. Vernizzi, Healthy theories beyond Horndeski, Phys. Rev. Lett. 114 (2015) 211101 [arXiv:1404.6495] [INSPIRE].

[13] J. Gleyzes, D. Langlois, F. Piazza and F. Vernizzi, Exploring gravitational theories beyond Horndeski, JCAP 02 (2015) 018 [arXiv: 1408.1952] [INSPIRE].

[14] H. Motohashi and T. Suyama, Third order equations of motion and the Ostrogradsky instability, Phys. Rev. D 91 (2015) 085009 [arXiv:1411.3721] [INSPIRE].

[15] D. Langlois and K. Noui, Degenerate higher derivative theories beyond Horndeski: evading the Ostrogradski instability, JCAP 02 (2016) 034 [arXiv:1510.06930] [INSPIRE].

[16] H. Motohashi, K. Noui, T. Suyama, M. Yamaguchi and D. Langlois, Healthy degenerate theories with higher derivatives, JCAP 07 (2016) 033 [arXiv:1603.09355] [INSPIRE].

[17] X. Gao, Unifying framework for scalar-tensor theories of gravity, Phys. Rev. D 90 (2014) 081501 [arXiv:1406.0822] [INSPIRE].

[18] J. Ben Achour, M. Crisostomi, K. Koyama, D. Langlois, K. Noui and G. Tasinato, Degenerate higher order scalar-tensor theories beyond Horndeski up to cubic order, JHEP 12 (2016) 100 [arXiv:1608.08135] [INSPIRE].

[19] N. Barnaby and N. Kamran, Dynamics with infinitely many derivatives: The Initial value problem, JHEP 02 (2008) 008 [arXiv:0709.3968] [INSPIRE].

[20] N. Barnaby and N. Kamran, Dynamics with Infinitely Many Derivatives: Variable Coefficient Equations, JHEP 12 (2008) 022 [arXiv:0809.4513] [INSPIRE].

[21] H. Motohashi, T. Suyama and M. Yamaguchi, Ghost-free theory with third-order time derivatives, J. Phys. Soc. Jap. 87 (2018) 063401 [arXiv:1711.08125] [INSPIRE].

[22] R. Klein and D. Roest, Exorcising the Ostrogradsky ghost in coupled systems, JHEP 07 (2016) 130 [arXiv: 1604.01719] [INSPIRE].

[23] R. Kimura, Y. Sakakihara and M. Yamaguchi, Ghost free systems with coexisting bosons and fermions, Phys. Rev. D 96 (2017) 044015 [arXiv:1704.02717] [INSPIRE].

[24] M. Crisostomi, K. Koyama and G. Tasinato, Extended Scalar-Tensor Theories of Gravity, JCAP 04 (2016) 044 [arXiv: 1602.03119] [INSPIRE].

[25] R. Kimura, A. Naruko and D. Yoshida, Extended vector-tensor theories, JCAP 01 (2017) 002 [arXiv: 1608.07066] [INSPIRE]. 
[26] M. Crisostomi, R. Klein and D. Roest, Higher Derivative Field Theories: Degeneracy Conditions and Classes, JHEP 06 (2017) 124 [arXiv: 1703.01623] [INSPIRE].

[27] M. Crisostomi, K. Noui, C. Charmousis and D. Langlois, Beyond Lovelock gravity: Higher derivative metric theories, Phys. Rev. D 97 (2018) 044034 [arXiv:1710.04531] [InSPIRE].

[28] G. Gabadadze, K. Hinterbichler, J. Khoury, D. Pirtskhalava and M. Trodden, A Covariant Master Theory for Novel Galilean Invariant Models and Massive Gravity, Phys. Rev. D 86 (2012) 124004 [arXiv:1208.5773] [inSPIRE].

[29] K. Takahashi, H. Motohashi, T. Suyama and T. Kobayashi, General invertible transformation and physical degrees of freedom, Phys. Rev. D 95 (2017) 084053 [arXiv: 1702.01849] [INSPIRE]. 